\title{
Cortactin Is a Regulator of Activity-Dependent Synaptic Plasticity Controlled by Wingless
}

\author{
-Daniel Alicea, ${ }^{1 \star}$ - Marizabeth Perez, ${ }^{1 \star}$ Carolina Maldonado, ${ }^{1,2}$ Carihann Dominicci-Cotto, ${ }^{1,2}$ and ${ }^{-B}$ Bruno Marie ${ }^{1,2}$ \\ ${ }^{1}$ Institute of Neurobiology and ${ }^{2}$ Department of Anatomy and Neurobiology, Medical Sciences Campus, University of Puerto Rico, San Juan, Puerto Rico \\ 00901
}

\begin{abstract}
Major signaling molecules initially characterized as key early developmental regulators are also essential for the plasticity of the nervous system. Previously, the Wingless (Wg)/Wnt pathway was shown to underlie the structural and electrophysiological changes during activity-dependent synaptic plasticity at the Drosophila neuromuscular junction. A challenge remains to understand how this signal mediates the cellular changes underlying this plasticity. Here, we focus on the actin regulator Cortactin, a major organizer of protrusion, membrane mobility, and invasiveness, and define its new role in synaptic plasticity. We show that Cortactin is present presynaptically and postsynaptically at the Drosophila NMJ and that it is a presynaptic regulator of rapid activity-dependent modifications in synaptic structure. Furthermore, animals lacking presynaptic Cortactin show a decrease in spontaneous release frequency, and presynaptic Cortactin is necessary for the rapid potentiation of spontaneous release frequency that takes place during activity-dependent plasticity. Most interestingly, Cortactin levels increase at stimulated synaptic terminals and this increase requires neuronal activity, de novo transcription and depends on $\mathrm{Wg} / \mathrm{Wnt}$ expression. Because it is not simply the presence of Cortactin in the presynaptic terminal but its increase that is necessary for the full range of activity-dependent plasticity, we conclude that it probably plays a direct and important role in the regulation of this process.
\end{abstract}

Key words: activity-dependent plasticity; miniature EPSP; NMJ; synaptic plasticity; Wingless/Wnt

\section{Significance Statement}

In the nervous system, changes in activity that lead to modifications in synaptic structure and function are referred to as synaptic plasticity and are thought to be the basis of learning and memory. The secreted Wingless/Wnt molecule is a potent regulator of synaptic plasticity in both vertebrates and invertebrates. Understanding the molecular mechanisms that underlie these plastic changes is a major gap in our knowledge. Here, we identify a presynaptic effector molecule of the Wingless/Wnt signal, Cortactin. We show that this molecule is a potent regulator of modifications in synaptic structure and is necessary for the electrophysiological changes taking place during synaptic plasticity.

\section{Introduction}

Identifying the molecular mechanisms by which neural activity leads to the modification of synaptic structure and function re-

\footnotetext{
Received April 26, 2016; revised Dec. 5, 2016; accepted Jan. 17, 2017.

Author contributions: D.A., M.P., C.M., C.D.-C., and B.M. designed research; D.A., M.P., C.M., C.D.-C., and B.M. performed research; D.A., M.P., C.M., C.D.-C., and B.M. analyzed data; B.M. wrote the paper.

This work was supported by National Institute of General Medical Sciences (NIGMS) Grant 1P20GM103642 (B.M., M.P.), NINDS Grant SC2NS077924 and NSF Human Resource Development Grant 1137725 (B.M., D.A), National Institute on Minority Health and Health Disparities Grant 8G12-MD007600 (Research Centers in Minority Institutions), and NIGMS Research Initiative for Scientific Enhancement Grants R25GM061838 (C.D., C.M.) and R25GM06115115 (M.P.). We thank Drs. Graeme Davis, Andrew Frank, and Pernille Rørth for providing fly strains; Dr. Shin Togashi for providing the anti-Cortactin antibody; and Dr. Jonathan Blagburn and Dr. Patrick Emery for their valuable comments on previous versions of this manuscript.

*D.A. and M.P. contributed equally to this work.

The authors declare no competing financial interests.

Correspondence should be addressed to Bruno Marie, Institute of Neurobiology, 201 Boulevard del Valle, San Juan, Puerto Rico 00901. E-mail: bruno.marie@upr.edu.
}

mains an essential challenge. Intense work has been performed isolating molecules and pathways that transduce the changes in intracellular $\mathrm{Ca}^{2+}$ concentration that result from neuronal activity (Hell, 2014; Cohen et al., 2015). It has recently become clear that major signaling molecules that were initially characterized as key early developmental regulators are also critical for the plasticity of the nervous system (Poon et al., 2013). Some of these molecules are involved in restructuring the synapse: for example, a lack of Netrin late in development provokes smaller dendritic spines in pyramidal neurons (Horn et al., 2013), while Wnt family members are involved in mediating activity-dependent dendritic arborization (Yu and Malenka, 2003; Rosso et al., 2005; Wayman et al., 2006). In both vertebrates and invertebrates, Wnt signaling regulates synaptic function (Koles and Budnik, 2012; 
Salinas, 2012). In Drosophila, the neuromuscular junction (NMJ) can be used as a model to assess the mechanisms involved in activity-dependent plasticity (Ataman et al., 2008). Upon repeated stimulation, the NMJ shows modifications in synaptic structure and function: new synaptic boutons are formed, and an increase in the frequency of miniature EPSPs (mEPSPs) is observed. Interestingly, this phenomenon depends on transcription, translation, and the activation of the Wnt signaling pathway (Ataman et al., 2008).

How does a change in Wnt signaling translate into morphological and physiological modifications? One candidate mechanism is via reorganization of the cytoskeleton, since actin polymerization is the major force behind several cellular processes such as cell adhesion, migration and division (Stricker et al., 2010). In addition, the importance of actin regulation in presynaptic assembly and in the formation of dendritic spines has been established (Bosch and Hayashi, 2012; Nelson et al., 2013). Previously, the activity of the actin regulator Cofilin was shown to be critical to the modification of synaptic structures at the NMJ (Piccioli and Littleton, 2014). Among the many other molecules involved in regulating actin dynamics, Cortactin (Cttn) is particularly interesting since it promotes actin polymerization and stabilizes branched actin structures after their formation (Uruno et al., 2001; Weaver et al., 2001, 2002; Goley and Welch, 2006). As such, it has been characterized as a major regulator of cell protrusion, membrane mobility and cancer invasiveness (Ammer and Weed, 2008; Kirkbride et al., 2011). In addition, previous studies on embryonic sensory axons have shown that NGF can lead to an increase of axonal Cttn expression to promote collateral branching and the emergence of filopodia (Spillane et al., 2012). Cttn also controls spine morphogenesis in an activity-dependent manner (Hering and Sheng, 2003; Iki et al., 2005; Chen and Hsueh, 2012; Lin et al., 2013), and a decrease in Cttn expression in the brain was linked to schizophrenia (Bhambhvani et al., 2016), a neuropathology associated with alterations in synaptic plasticity (Crabtree and Gogos, 2014). This makes Cttn an ideal candidate to control rapid activity-dependent synaptic plasticity at the NMJ.

Here, we show that Cttn is present at the NMJ and that it is required presynaptically for the morphological and electrophysiological modifications associated with synaptic plasticity. We find that stimulated synapses show a 100-200\% increase in levels of Cttn protein. This increase in synaptic Cttn after stimulation is dependent on de novo transcription and $\mathrm{Wg} / \mathrm{Wnt}$ expression and is essential for synaptic plasticity to take place. We propose a model where $\mathrm{Wg} / \mathrm{Wnt}$ signaling controls the increase of Cttn expression to regulate rapid activity-dependent synaptic plasticity.

\section{Materials and Methods}

Genetics. Animals of either sex were used throughout the study. We used the following null alleles: $c t t n^{M 7}$ (a kind gift from Dr. P. Rørth; Somogyi and Rørth, 2004), $\mathrm{ctt}^{6 A 2}$ [Bloomington Drosophila stock center (BDSC), stock \#9367], and Df(cttn) [DF(3R)Exel6272; BDSC, stock \#7739]. The $w g^{t s}$ is $w g^{I-12}$ (BDSC stock \#7000). We used the paralytic allele para ${ }^{\text {tsl }}$ (Ganetzky, 1984). The synaptotagmin 1 alleles were Syt $1^{A D 4}$ (DiAntonio and Schwarz, 1994) and Syt1 ${ }^{\text {N13 }}$ (Littleton et al., 1994; BDSC stock \#39667). We used the Gal4/UAS system (Brand and Perrimon, 1993) to express RNA interference (RNAi) constructs or overexpress genes in either neuron or muscle by using either the elav ${ }^{\mathrm{Cl} 55}$-Gal4 or MHC-Gal4 driver in conjunction with UAS-CttnRNAi [ $y^{1} s c^{*} v^{1}$; P(TRiP.HMS00658), BDSC, stock \#32871], Tub-Gal80 ${ }^{t s}$ (BDSC, stock \#7108), UAS-Fz2-RNAi (BDSC, stock \#31390), UAS-wg-HA (BDSC, stock \#5918), and UAS-cttn.

Stimulation protocol. Our stimulation protocol was adapted from Ataman et al., 2008. It consists of five stages of alternating stimulation and rest periods. The first three stages are composed of a 2 min stimulation step followed by a $15 \mathrm{~min}$ rest period. The fourth stage is composed of a 4 min stimulation step followed by a 15 min rest, and the fifth and final stage is composed of a 6 min stimulation step followed by a 14 min rest. This $90 \mathrm{~min}$ protocol is used throughout the manuscript unless otherwise stated. We also shortened and lengthened the last 14 min rest step to 4 and $44 \mathrm{~min}$ to make stimulation protocols of 80 and $120 \mathrm{~min}$ long, respectively. Both these stimulation protocols were used in time course experiments (see Fig. $2 A-C$ ). The 120 min stimulation protocol was used in the electrophysiology experiments (see Figs. 6, 7). The preparation was stimulated by application of Haemolymph-like HL3 saline $(70 \mathrm{~mm} \mathrm{NaCl}$, $10 \mathrm{~mm}$ NaHCO3, $115 \mathrm{~mm}$ sucrose, $5 \mathrm{~mm}$ trehalose, 5 mM HEPES, $10 \mathrm{~mm}$ $\mathrm{MgCl} 2$ ) containing $90 \mathrm{~mm} \mathrm{KCl}$ and $1.5 \mathrm{mM} \mathrm{CaCl}_{2}$, while rest periods consisted of application of HL3 saline containing $5 \mathrm{~mm} \mathrm{KCl}$ and $0.1 \mathrm{~mm}$ $\mathrm{CaCl}_{2}$.

Immunohistochemistry. Before using the polyclonal anti-Cortactin antibody (Katsube et al., 1998), we preincubated the working dilution (1:50) with preparations devoid of the Cortactin protein (8 to 12 Cortactin null mutants, $\mathrm{cttn}^{M 7}$ ) for $2 \mathrm{~d}$ at $4^{\circ} \mathrm{C}$. After the repeated stimulation protocol was performed, preparations were fixed for $15 \mathrm{~min}$ at room temperature in a solution of $4 \%$ paraformaldehyde in PBS. Primary antibodies anti-Dlg (1/20; Budnik et al., 1996) and anti-Cortactin (1:50) were applied overnight at $4^{\circ} \mathrm{C}$. Anti-Hrp (1:300; Jan and Jan, 1982), Cy3-conjugated AffiniPure goat anti-horseradish peroxidase (Jackson ImmunoResearch), and secondary antibodies (1:300; Alexa Fluor 488conjugated AffiniPure goat anti-mouse or anti-rabbit IgG, Jackson ImmunoResearch; Cascade Blue goat anti-mouse, Invitrogen) were applied for $1 \mathrm{~h}$ at room temperature as described previously (Marie et al., 2010; Maldonado et al., 2013).

Quantification of ghost boutons. The stimulation protocol was performed as described above. For each genotype or condition tested, a set of controls $\left(w^{1118}\right)$ was run in parallel to account for the potential variation in our experimental manipulations. Ghost boutons were defined as positive for anti-HRP and negative for anti-Dlg immunoreactivity. Counting was performed on NMJs at muscle $6 / 7$ on segment A3 and averaged across same conditions and/or genotype. We used a Nikon Eclipse 80i microscope at a magnification of $400 \times$ to carry out these observations.

Quantification of synaptic proteins. For comparison of fluorescence intensities, the preparations were processed and imaged identically using a Zeiss LSM 5 Pascal confocal microscope with a $63 \times, 1.4$ numerical aperture objective. Individual entire muscle $4 \mathrm{NMJs}$ in abdominal segment A3 were optically sectioned, and a $2 \mathrm{D}$ maximum intensity $Z$ projection was made. The entire synaptic area was selected using ImageJ software (https://imagej. nih.gov/ij/) and the average fluorescence calculated. A muscle area devoid of synaptic boutons was also selected and quantified to establish the background intensity level. The fluorescence intensity values represent the difference between the synaptic intensity and muscle intensity $(\Delta F)$ over the intensity of the muscle $(F)$ normalized to wild-type values (Marie et al., 2004, 2010; Maldonado et al., 2013) .

NMJ electrophysiology. Whole-muscle recordings were performed on muscle 6 in abdominal segment A3 using sharp microelectrodes (10-16 $\mathrm{M} \Omega$ ) as described previously (Maldonado et al., 2013). Only the recordings with resting membrane potentials exceeding $-60 \mathrm{mV}$ and with input resistances $>5 \mathrm{M} \Omega$ were selected for the analysis. The average mEPSP amplitude was quantified by averaging the amplitude of 100-200 individual sequential spontaneous mEPSP events per NMJ, using Mini Analysis software (Synaptosoft). Measurements were carried out $120 \mathrm{~min}$ after the start of the stimulation protocol.

Statistical treatment. We first assessed whether data conformed to a normal distribution by performing a Shapiro-Wilk normality test. When the Shapiro-Wilk normality test was low ( $p<0.0001)$, we ran a nonparametric Kruskal-Wallis test with a post hoc Dunn's multiple comparisons test (Fig. 1E). In the other cases, we ran a parametric ANOVA. The post hoc Dunnett correction test was applied when multiple comparisons were carried out against a control value (these comparisons are indicated with asterisks over the bars in the figures), while the post hoc Tukey correction test was used for multiple comparisons between data sets (these comparisons are indicated with brackets and asterisks in the fig- 

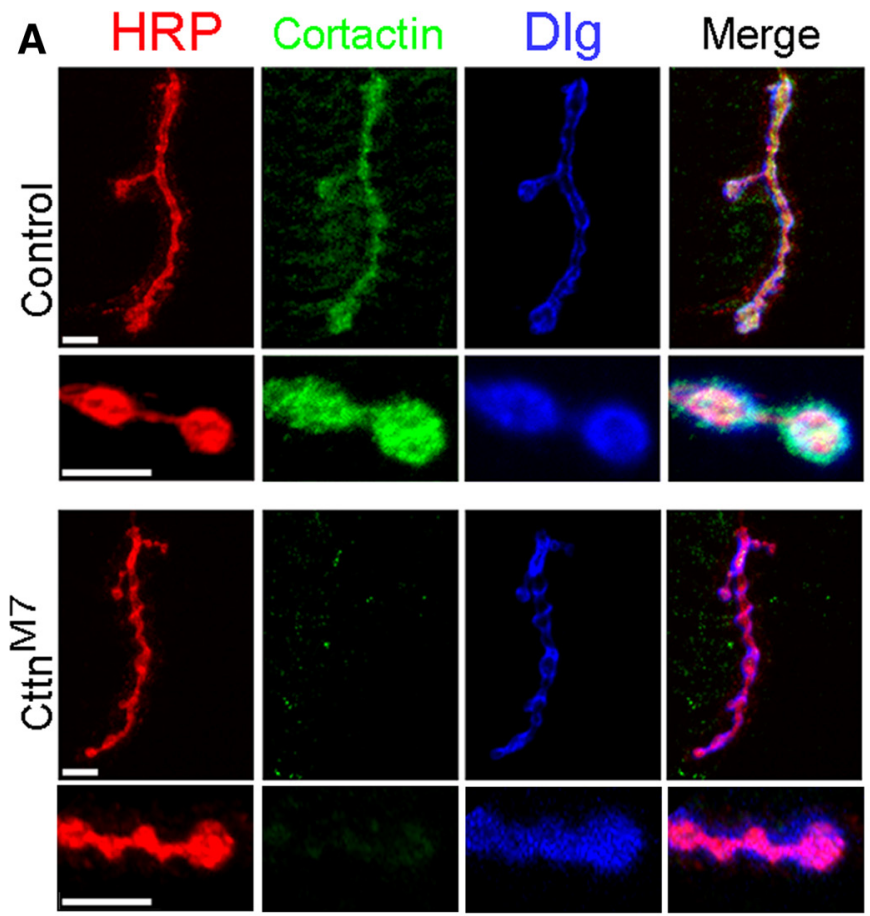

B
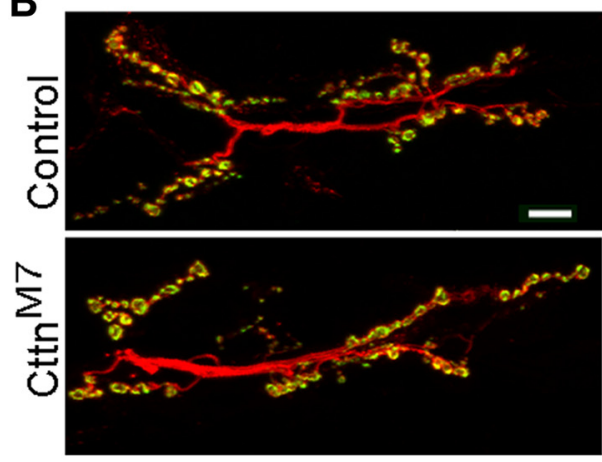

Synaptic growth (\% of control value) 120. Third Instar larvae
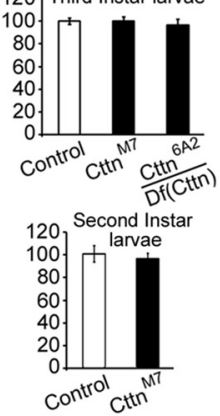

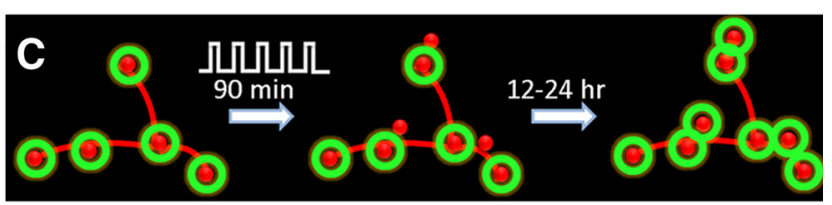

D
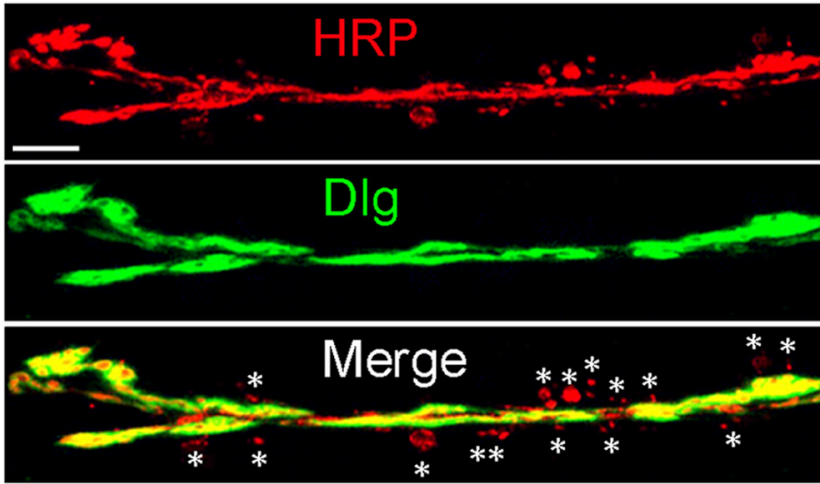

E

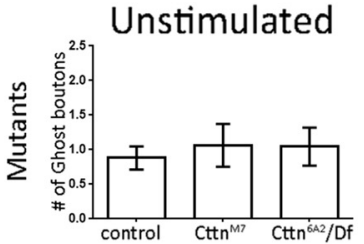

F
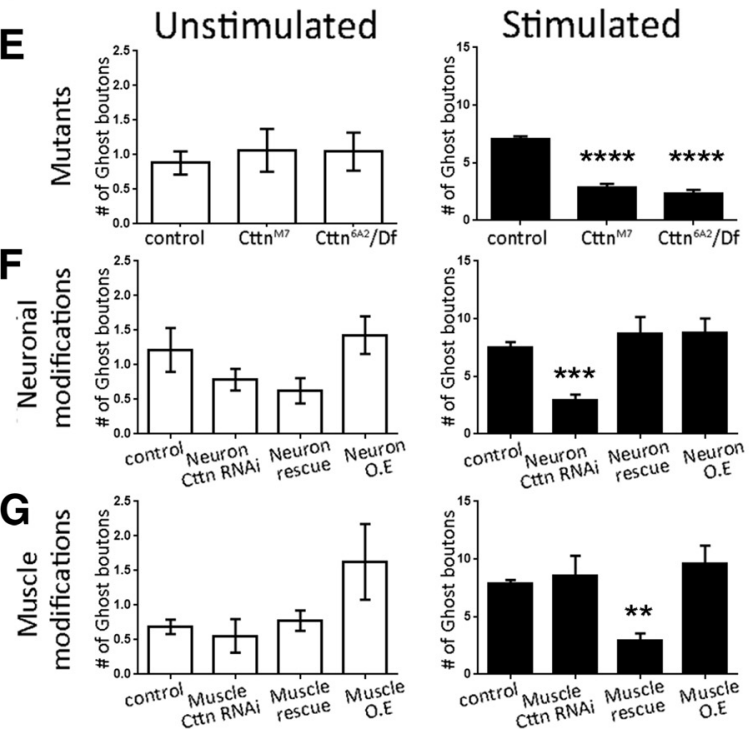

Figure 1. Cortactin, which is present at the NMJ, is not required for synaptic growth but is an essential regulator of activity-dependent synaptic growth. $\boldsymbol{A}$, Representative muscle 4 synapses in control and $\mathrm{Cttn}^{M 7}$ mutant animals showing immunostaining for the presynaptic membrane marker HRP (red), Cortactin (green), and the postsynaptic marker Dlg (blue), showing the complete absence of Cortactin in the mutant. An example of a couple of control synaptic boutons is also provided showing that Cttn is present presynaptically and postsynaptically. $\boldsymbol{B}$, Representative muscle 6/7 synapses and quantification of synaptic growth (mean number of synaptic boutons at muscle 6/7 segment A3), showing that (ttn mutants do not show altered synaptic growth at two different developmental stages. C, A schematic representation of a synapse subjected to repeated stimulation showing a number of ghost boutons (presynaptic red staining only) that will show postsynaptic differentiation (green) 12 to $24 \mathrm{~h}$ later. D, A representative synapse 90 min after repeated stimulation showing anti-HRP (red, presynaptic) and anti-Dlg (green, postsynaptic) immunoreactivity, displaying ghost boutons with no Dlg staining (asterisks). Scale bars: $10 \mu \mathrm{m} . \boldsymbol{E}$, Quantification of the number of ghost boutons in unstimulated and stimulated preparations in control ( $w^{-}$) and mutant [ $c t n^{M 7}$ and $\left.c t t n^{6 A 2} / D f(c t t n)\right]$ synapses $(n=59,16,22,125,35,13)$. $F$, Quantification of the number of ghost boutons in unstimulated and stimulated preparations in control (the neuronal driver elav-Gal4/+), neuron cttn RNAi (elav-Gal 4/+; UAS-cttn ${ }^{R N A i}$ ), cttn neuronal rescue [elav-Gal4/+; UAS-cttn/+; cttn $\left.{ }^{6 A 2} / D f(c t t n)\right]$, and cttn neuronal overexpression (elav-Gal4/+; UAS$\mathrm{cttn} /+$ ) synapses ( $n=14,14,8,14,32,14,10,20)$. G, Quantification of the number of ghost boutons in unstimulated and stimulated preparations in control (the muscle driver MHC-Gal4/+), muscle $c t t n$ RNAi $\left(M H C-G a l 4 / U A S-c t t n^{R N A i}\right)$, cttn muscle rescue [UAS-cttn/+;MHC-Gal4, Df(cttn)/cttn ${ }^{6 A 2}$ ], and cttn overexpression (UAS-cttn/+; MHC-Gal4/+) synapses ( $n=32,9,9,16,19,9,10$, 14). ${ }^{* *} p<0.01 ;{ }^{* * *} p<0.001 ;{ }^{* * *} p<0.0001$ [Kruskal-Wallis test with Dunn's multiple comparisons $(\boldsymbol{E})$ or ANOVA with post hoc Dunnett test $(\boldsymbol{F}, \boldsymbol{G})$ ]. Data represent mean \pm SEM.

ures). When only two data sets were compared, we performed an unpaired, two-tailed $t$ test.

\section{Results}

The actin regulator Cortactin is present presynaptically and postsynaptically at the NMJ but does not influence synaptic morphology

Because cytoskeletal rearrangements and actin dynamics are essential for the establishment of a synapse, we first investigated whether the actin regulator Cttn is present at the NMJ. We focused on Cttn because of its documented function in the formation of dendritic spines (Hering and Sheng, 2003) and its role in the emergence of filopodia and axonal collateral branches during development (Spillane et al., 2012). We were able to detect Cttn immunolabeling at control NMJs, while no labeling was seen in the $\mathrm{cttn}^{M 7}$ null mutant (Somogyi and Rørth, 2004) (Fig. 1A). Coimmunolabeling with the presynaptic membrane marker HRP and the postsynaptic marker Dlg (the PSD-95 homolog; Budnik et al., 1996) showed that Cttn is present both presynaptically and postsynaptically at the NMJ (Fig. $1 A)$. We then asked whether Cttn could have a role in synaptic development and growth. In cortactin mutants, we did not observe any axonal misrouting (data not shown) or anomalies in synaptic morphology. Indeed, we used three different cortactin null alleles- 
$c t n^{M 7}, c t t n^{6 A 2}$, and $D f(c t t n)$ - and assessed the $c t t n^{M 7}$ homozygotes as well as $\mathrm{cttn}^{6 A 2} / \mathrm{Df}(\mathrm{cttn})$ animals. $C t t n^{M 7}$ and $C t t n^{6 A 2} / D f(C t t n) \mathrm{mu}-$ tants presented the same synaptic growth (number of synaptic boutons in third instar larvae; Fig. 1B) as control animals. We also examined synaptic growth earlier during development at the second instar stage (Fig. 1B). At this stage also, synaptic growth is not affected by the lack of Cttn. We conclude that synaptic growth or its kinetics are unaffected in cttn mutant animals.

\section{Presynaptic Cortactin is a regulator of activity-dependent modification in synaptic structure}

Although Cttn did not seem to have a role in synaptic growth or development, it is possible that it could regulate the structural modifications that occur during activity-dependent synaptic plasticity. To investigate this, we used a previously described experimental paradigm at the Drosophila NMJ, in which repeated stimulation provokes modifications in synaptic structure (Ataman et al., 2008). These de novo outgrowths, which will show clustering of glutamate receptors at 12 to $24 \mathrm{~h}$ after stimulation, show presynaptic membrane only $90 \mathrm{~min}$ after the start of the stimulation protocol (Fig. 1C,D). These structures, devoid of postsynaptic differentiation (Fig. 1C,D), are named ghost boutons (Ataman et al., 2008). Here, we count the number of these ghost boutons (showing presynaptic anti-HRP staining only) at the synapse $90 \mathrm{~min}$ after repeated stimulation to quantify this activity-dependent plasticity. In agreement with previously published data (Ataman et al., 2008), control NMJs exposed to the stimulus protocol showed an average of $7 \pm 0.3$ ghost boutons (for $w^{-}$animals; Fig. $1 D, E$ ), while unstimulated synapses showed only $0.9 \pm 0.2$ ghost boutons (Fig. $1 E$ ).

To ask whether Cttn was necessary for activity-dependent modification of synaptic structure, we assessed the $\mathrm{cttn}^{M 7}$ homozygotes as well as $\mathrm{cttn}^{6 \mathrm{~A} 2} / \mathrm{Df}(\mathrm{cttn})$ animals for the presence of ghost boutons after repeated stimulation (Fig. 1E). In both allelic combinations, the absence of $\mathrm{Cttn}$ rendered the synapse less sensitive to this treatment. Indeed, the number of ghost boutons after stimulation was $2.8 \pm 0.3$ in $\mathrm{cttn}^{\mathrm{M7}}$ and $2.3 \pm 0.3$ in $\mathrm{cttn}^{6 \mathrm{~A} 2}$ / $D f(c t t n)$ animals, reductions of 60 and $67 \%$, respectively (Fig. 1E; $p<0.0001)$. Hence, $\mathrm{Cttn}$ is a potent regulator of activitydependent modification of synaptic structure. We then asked whether this effect could be primarily attributed to neuronal or muscle Cttn. To do so, we used transgenic animals expressing RNAi against $\mathrm{Cttn}$ in either neuron (Fig. $1 F$ ) or muscle $(G)$. The stimulated animals expressing neuronal $c t t n$ RNAi showed a reduction of $61 \%$ in the number of ghost boutons when compared to stimulated controls (Fig. $1 F ; p=0.0007$ ), similar to what we observed in $\mathrm{ctth}^{M 7}$ and $c t t n^{6 A 2} / \mathrm{Df}(\mathrm{cttn})$ animals (Fig. 1E) and demonstrating the efficacy of the $c t t n$ RNAi construct. In contrast, animals expressing $\mathrm{cttn}$ RNAi in the muscle still showed the appearance of ghost boutons after repeated stimulation; these animals were not significantly different from the control strains $(p=0.66$; Fig. $1 G)$. To investigate this further, we performed rescue experiments where Cttn cDNA was driven by a neuronal

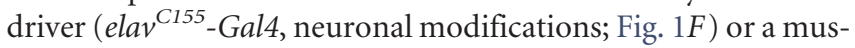
cle driver (MHC-Gal4, muscle modifications; Fig. $1 G$ ) in an otherwise Cortactin mutant fly [Cort $\left.{ }^{6 A 2} / D f(c t t n)\right]$. The animals rescued in the neurons showed a normal number of ghost boutons after repeated stimulation; controls and rescues were not significantly different $(p=0.41$; Fig. $1 F)$. In contrast, the animals expressing Cttn in the muscle behaved as $\mathrm{cttn}$ mutants; they showed a significant decrease compared to controls $(p=0.008$;
Fig. 1G). We conclude that it is the neuronal Cttn that is a potent regulator for the establishment of the de novo synaptic boutons after repeated stimulation. Interestingly, the fact that $\mathrm{Cttn}$ is not necessary for synaptic growth during development (or its absence can be compensated for) suggests that the deficiency in synaptic structural plasticity observed in the $c t t n$ mutants is not merely a consequence of impaired synaptic growth, but might instead imply that Cttn functions as a modulator of plasticity-related growth.

\section{Cortactin is present in ghost boutons, and Cortactin levels increase at stimulated synaptic terminals}

Because Cttn is present at synaptic terminals and is important for activity-dependent changes in synaptic structure, we asked whether it is present in the newly formed ghost boutons. To this effect, we stimulated the animals using a 90-min-long protocol (see Materials and Methods) and immunolabeled synapses for HRP, Dlg, and Cttn. We then asked whether Cttn immunolabeling was detectable in ghost boutons (defined as presynaptic HRP immunostaining only, with no postsynaptic $\mathrm{Dlg}$ ). We examined 104 ghost boutons from 12 stimulated synapses and found that $64 \%$ of the ghost boutons presented detectable Cttn expression (Fig. 2A). This is consistent with it playing a critical role in the formation of ghost boutons, but perhaps not persisting throughout their lifespan. To test this possibility, we shortened ( $80 \mathrm{~min}$, $n=12$ ) or lengthened (120 min, $n=11$ ) the last rest step of our stimulation protocol and asked whether this impacted the amount of ghost boutons produced or the percentage of ghost boutons containing $\mathrm{Cttn}$. We found no difference in the amount of ghost boutons produced at these different times (Fig. 2A; $p=0.19$ ). In contrast, the expression of Cttn in ghost boutons differed greatly at $80 \mathrm{~min}$. It was present in $93 \%$ of the ghost boutons observed (Fig. $2 A ; p=0.0004$ ). This result strongly suggests that Cttn is predominantly involved in the early stages of ghost bouton formation.

We also assessed the abundance of Cttn within entire synaptic terminals of muscle 4 at rest or after stimulation (Fig. $2 B-D$ ), using previously established methods for quantification of synaptic immunostaining (Marie et al., 2004, 2010; Maldonado et al., 2013). We used the presynaptic marker HRP to define a synaptic region of interest and quantified Cttn fluorescence intensity within this region (Fig. 2B,C). To our surprise, we observed a large increase in synaptic Cttn after repeated stimulation (Fig. $2 B, D)$. Indeed, at stimulated synapses, Cttn immunostaining levels were $242 \%$ of the unstimulated values. However, in the same synapses, the level of anti-HRP fluorescence remained unchanged after stimulation (102\%) compared to unstimulated synapses (Fig. 2B,D). In addition, this doubling of Cttn levels after repeated stimulation was observed at other neuromuscular synapses. For example, stimulated muscles $6 / 7$ synapses showed Cttn levels of 201\% compared to unstimulated synapses $(n=15$; data not shown). We also labeled the synapse with the postsynaptic marker Dlg (Fig. 2B) and selected the synaptic area containing Dlg staining and excluding HRP (postsynapse only; Fig. 2C). This compartment also showed a significant increase in Cttn immunostaining (Fig. 2D); after stimulation, it reached $236 \%$ of control preparations $(n=22$ and $21 ; p=0.0002)$. These results show that, upon stimulation, the level of synaptic Cttn is increased and strongly suggest that it is part of the cellular machinery activated during activity-dependent synaptic plasticity. 

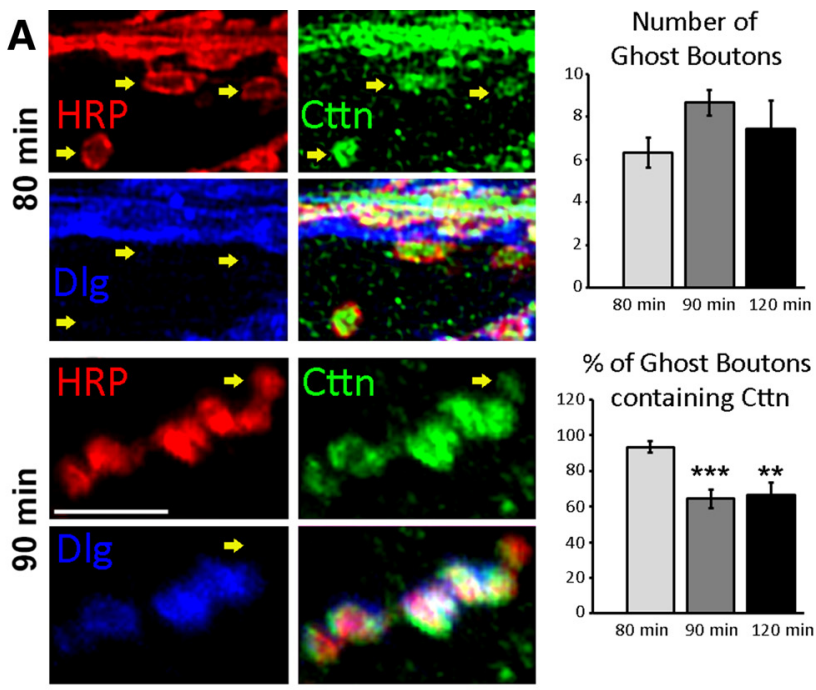

$\%$ of Ghost Boutons

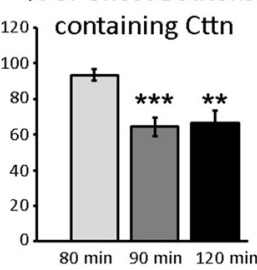

B
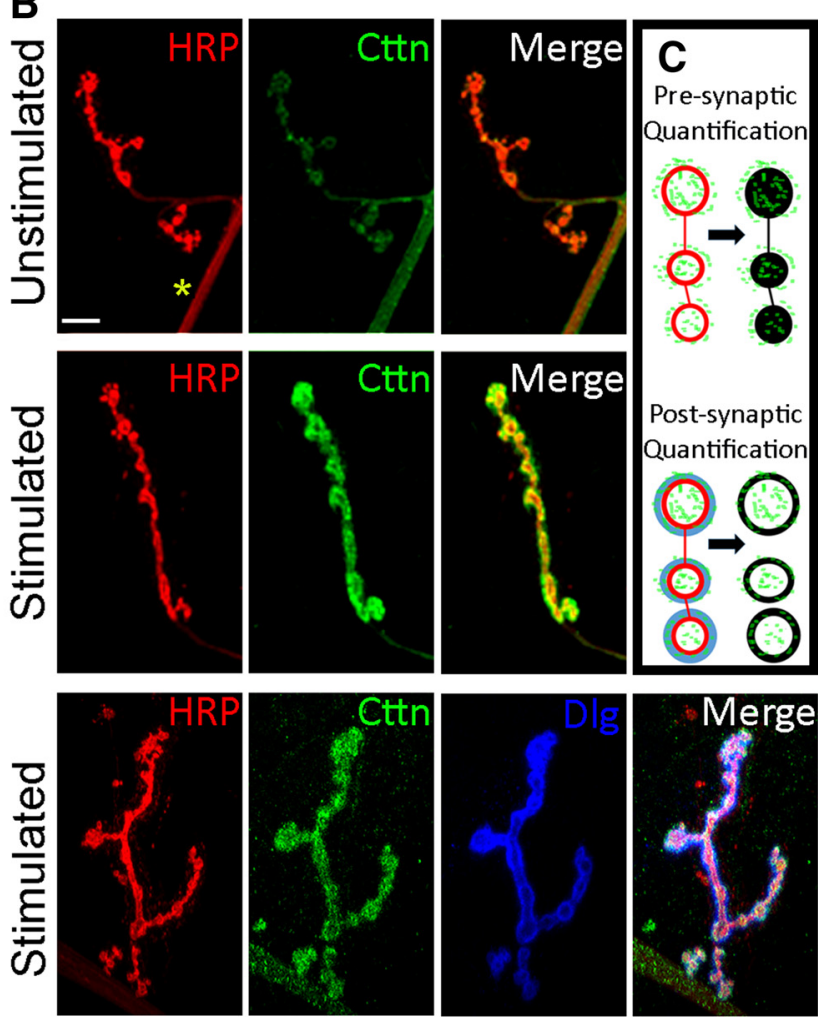

D Pre-synaptic Staining Intensity $(\Delta F / F)$
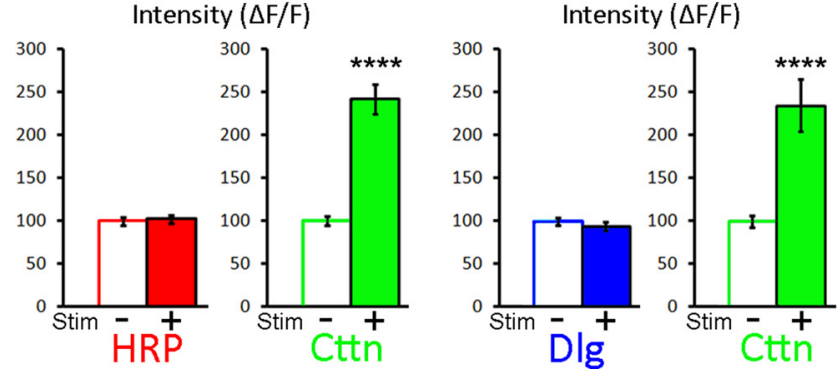

Figure 2. Cortactin is present in ghost boutons, and its abundance at the synapse is more than doubled following repeated stimulations. $A$, A representative series of synaptic boutons at muscle 6/7 showing anti-HRP, anti-Dlg, and anti-Cortactin immunofluorescence during 80 and $90 \mathrm{~min}$ stimulation protocols. The arrows point at ghost boutons containing presynaptic Cortactin. On the right, quantifications show that the duration of our stimulation protocols affects the percentage of (ttn-positive ghost boutons (bottom) without affecting the total number of
The increase of Cortactin at stimulated synapses promotes activity-dependent modifications in synaptic structure We hypothesized that the increased amount of Cttn at stimulated synapses (and not its mere presence) was promoting activitydependent synaptic plasticity. To test this, we used different genetic conditions that result in different amounts of synaptic Cttn after repeated stimulation. We then asked whether these different amounts of Cttn had an influence on the magnitude of the modifications in synaptic structures. We first analyzed the animals expressing cttn RNAi in neurons (Fig. 3C-E). In these animals, the presynaptic Cttn immunostaining intensity before stimulation was $41 \%$ of unstimulated control levels, and after stimulation it increased to only $61 \%$, instead of to $278 \%$ as in controls. This led to the appearance of an average of $2.9 \pm 0.5$ ghost boutons (Fig. 3E), a number comparable to that observed in cttn null mutants (Fig. 1E). We concluded that this amount of Cttn is not sufficient to mediate the full morphological modifications occurring during activity-dependent synaptic plasticity. We then turned to the heterozygote combination ( $\mathrm{cort}^{6 A 2} /+$; Fig. $3 B, D, E)$, which should have approximately half the normal "dose" of the protein. Our quantification of synaptic Cttn levels in heterozygote animals showed that there was indeed $49 \%$ of control Cttn immunostaining intensity at rest, and this increased to $136 \%$ after stimulation (Fig. $3 D$ ). At this level of synaptic Cttn, the number of ghost boutons at the synapse was $4.5 \pm 0.7$, a number significantly smaller than the number of ghost boutons at stimulated control synapses $(8.5 \pm 0.6)$ containing a level of Cttn of $278 \%$ (Fig. $3 A, D ; p<0.0001$ ), and not significantly greater than the number of boutons at stimulated $c t t n$ RNAi synapses $(p=0.139)$. Hence, the amount of Cttn at rest (or even a $36 \%$ increase in it) is not sufficient to promote the Cttndependent component of the activity-dependent synaptic plasticity. We therefore conclude that the increased amount of Cttn at stimulated synapses is an essential part of the activity-dependent modifications in synaptic structure. It is interesting to note that in both the control and heterozygote animals, the increase in synaptic Cttn immunostaining between unstimulated and stimulated synapses is identical (2.8-fold), suggesting that it is the absolute amount, rather than the relative increase, in presynaptic Cttn that determines the magnitude of the structural modifications at the NMJ. The fact that there seems to be a linear relationship (Fig. 3F) between the amount of Cttn at the synapse and the number of ghost boutons after stimulation strengthens this hypothesis.

We then wanted to ask whether increased Cttn was sufficient to induce ghost boutons. To do so, we quantified synapses from animals that overexpressed Cttn in neurons or muscles. In both cases, the numbers of ghost boutons in unstimulated conditions were not different from controls $(p>0.99$ and $p=0.62$; Fig. $1 E)$. In addition, we quantified the intensity of presynaptic Cttn in

ghost boutons (top). $n=12,12,11 . \boldsymbol{B}$, Representative unstimulated and stimulated synapses at muscle 4 show that presynaptic and postsynaptic Cortactin levels are increased upon stimulation while anti-HRP and anti-Dlg levels stay constant. A star identifies a nerve. C, Schematic representation showing that the marker HRP is used to mask the presynaptic area for quantification, while the postsynaptic area is defined by the exclusion of HRP and the masking of Dlg marker. $\boldsymbol{D}$, Quantification of the presynaptic staining intensity shows anti-HRP and antiCortactin fluorescence intensity at unstimulated and stimulated synapses $(n=15,11,15,11)$. Quantification of the postsynaptic staining intensity shows anti-Dlg and anti-Cortactin fluorescence intensity at unstimulated and stimulated synapses $(n=22,21,22,21) .{ }^{* *} p<0.01$; ${ }^{* * *} p<0.001 ;{ }^{* * * *} p<0.0001$ [ANOVA with post hoc Dunnett test $(\boldsymbol{A})$ or $t$ test $(\mathbf{G})$ ]. Data represent mean \pm SEM. Scale bars: $10 \mu \mathrm{m}$. 

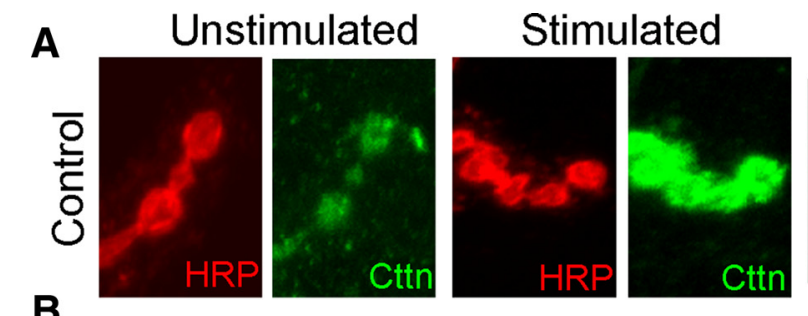

\section{Ghost boutons at Stimulated Synapses}
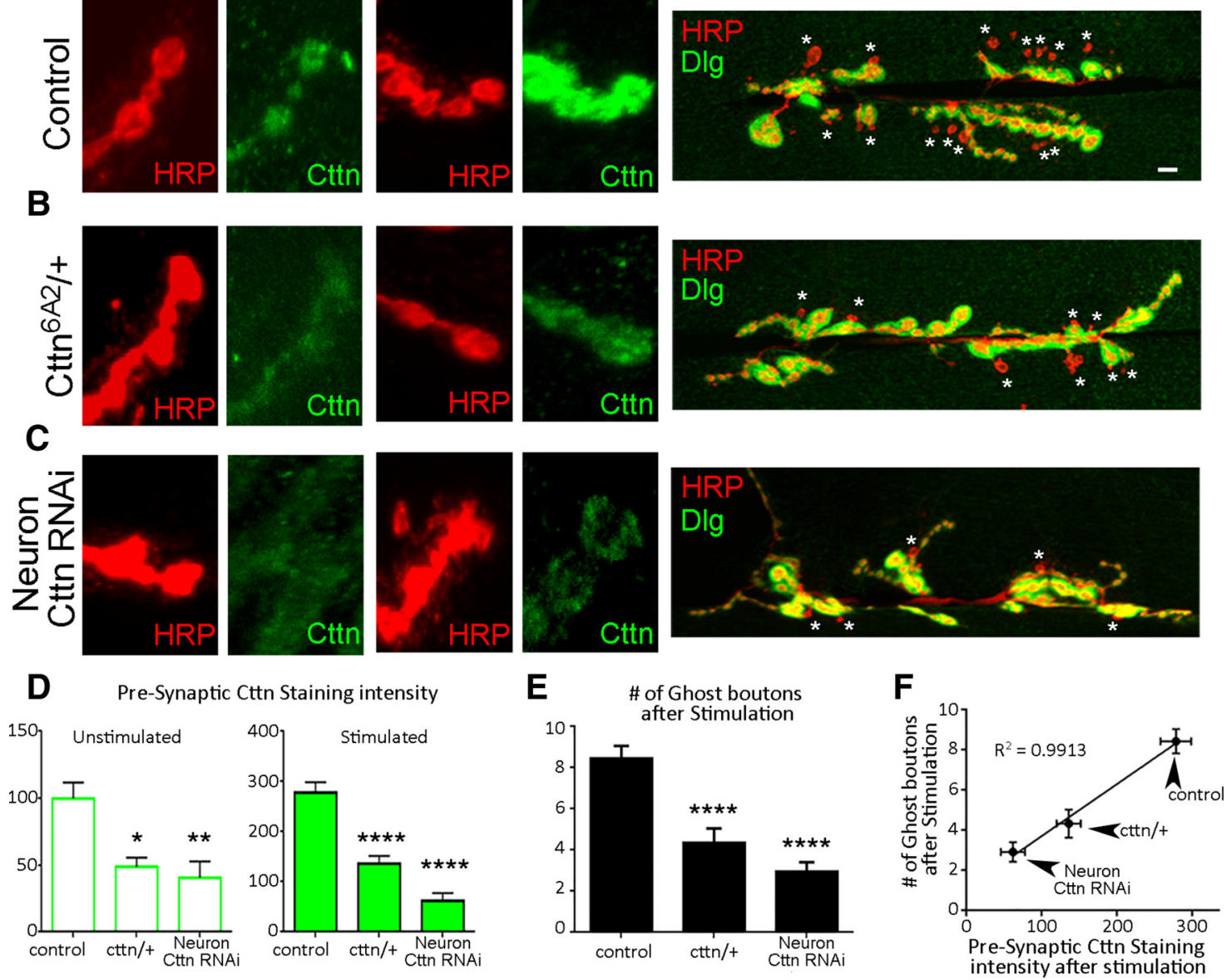

Figure 3. The increase of $\mathrm{Cttn}$ after stimulation is essential to the full expression of activity-dependent synaptic plasticity. $A-C$, Left, Representative synaptic boutons of unstimulated and stimulated preparations showing the membrane marker anti-HRP and Cortactin in controls $(\boldsymbol{A}), \mathrm{cttn}{ }^{6 \mathrm{~A} 2} /+(\boldsymbol{B})$, and animals expressing neuronal $\mathrm{cttn}$ RNAi $(\boldsymbol{C})$. Note that stimulated animals expressing neuronal cttn RNAi show an increase in postsynaptic Cttn only. Right, Representative muscle 6/7 synapse showing ghost bouton formation after stimulation (asterisks). D, Quantification of the amount of presynaptic $\mathrm{Cttn}$ immunostaining ( $\Delta F / F)$ presented as a percentage of unstimulated control level in unstimulated and stimulated animals $(n=21,7,10,23,12,11)$. $\boldsymbol{E}$, Number of ghost boutons $(n=14,16,14,20,20,14)$ after stimulation in control, heterozygote mutant, and neuronal cttn RNAi. $\boldsymbol{F}$, Linear relationship between Cttn staining intensity and ghost boutons formation after stimulation. A significant increase between resting and stimulated conditions is indicated. ${ }^{*} p<0.05 ;{ }^{* *} p<0.01 ;{ }^{* * *} p<0.0001$ (ANOVA with post hoc Dunnett test). Data represent mean \pm SEM. Scale bar: $10 \mu \mathrm{m}$.

neuronal overexpressers. These animals showed synapses with an immunostaining intensity of $185 \%$ (data not shown) compared to unstimulated controls. Because this amount of Cttn is comparable to the amount found in stimulated controls, but is unable to elicit ghost boutons, we conclude that Cttn overexpression alone is not sufficient to induce morphological modifications at the NMJ.

The increase of synaptic Cortactin during repeated stimulation requires de novo transcription

Since rapid activity-dependent plasticity has been shown to require de novo transcription (Ataman et al., 2008), we asked whether the increase of Cttn observed after repeated stimulation also required this process. We performed the repeated stimulation protocol in the presence of the transcription inhibitor actinomycin D (5 mM) and assayed the amount of synaptic Cttn (Fig. $4 A-E)$. The amount of Cttn at synapses where de novo transcription was inhibited was greatly reduced. Indeed, the amount of Cttn protein present at synapses treated with actinomycin D was only $36 \%$ of that in control untreated synapses (Fig. $4 A, C, E$ ), suggesting that synaptic Cttn is fairly unstable and that de novo transcription is required to replenish its pool at resting synapses. In addition, when synapses were stimulated in the presence of the transcription inhibitor, no increase of Cttn was observed. The amount of Cttn at these synapses was 35\% of that in control unstimulated and untreated synapses (Fig. $4 D, E$ ). This shows that de novo transcription is required for the increase of synaptic Cttn after repeated stimulation. It is interesting to note that the immunofluorescence associated with the anti-HRP antibody did not decrease after $90 \mathrm{~min}$ in presence of actinomycin D. Indeed, there was a significant increase (38\%) in anti-HRP immunoreactivity in synapses at rest in the presence of actinomycin D. Because the anti-HRP antibody recognizes an epitope present on several presynaptic membrane proteins (Snow et al., 1987; Katz et al., 1988), it is difficult to interpret the meaning of this increase. Nevertheless, this result shows that the decrease of Cttn in the presence of transcription inhibitor does not reflect a generalized decrease in synaptic proteins.

The dynamic activity-dependent synaptic expression of Cttn makes it suitable for a role as an instructive molecular switch. Indeed, Cortactin synaptic levels can more than double in 90 min, while its synaptic stability appears to be relatively low, allowing for repeated turning on and off over a short timescale. Because Cttn has been involved in spine plasticity (Hering and Sheng, 2003) and in the production of collateral branching and 


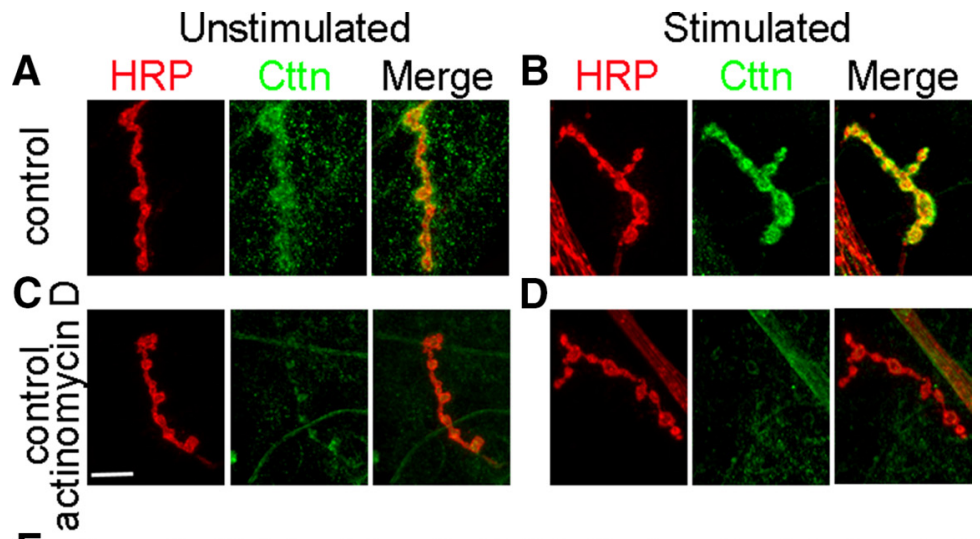

E Synaptic Staining Intensity $(\Delta F / F)-\%$ of unstimulated control

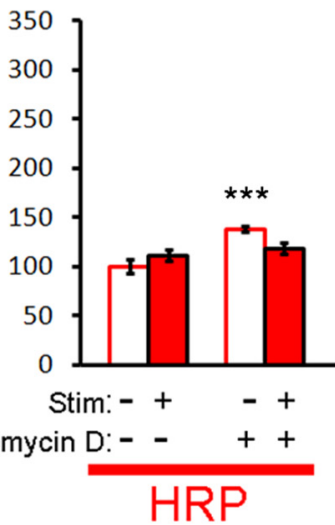

F Synaptic Staining Intensity $(\Delta \mathrm{F} / \mathrm{F})$ (\% of unstimulated control)

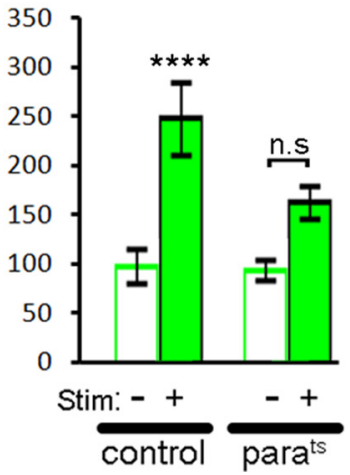

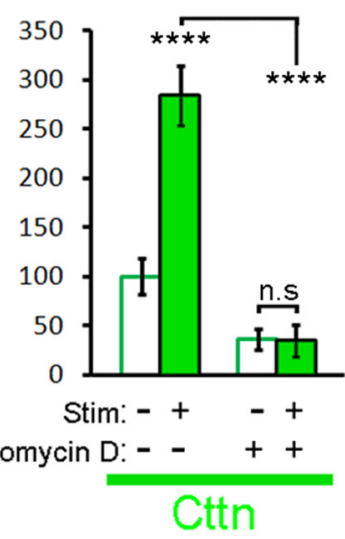

G Synaptic Staining Intensity $(\Delta \mathrm{F} / \mathrm{F})$ (\% of unstimulated control)

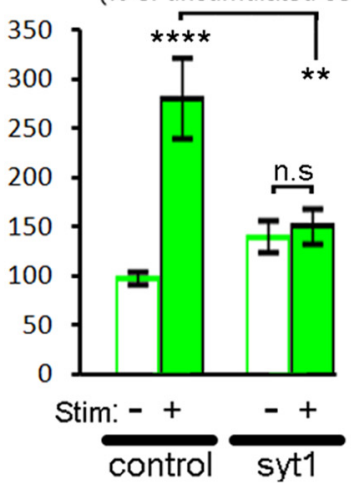

Figure 4. The increase in synaptic Cortactin after repeated stimulation requires de novo transcription. $A-D$, Synapses in control conditions $(\boldsymbol{A}, \boldsymbol{B})$ or in the presence of actinomycin $\mathrm{D}(\boldsymbol{C}, \boldsymbol{D})$, at rest $(\boldsymbol{A}, \boldsymbol{C})$ or after repeated stimulations $(\boldsymbol{B}, \boldsymbol{D})$. $\boldsymbol{E}$, Quantification of HRP and $C t$ tn fluorescence intensity at synapses in muscle 4 in the presence or absence of actinomycin $D$ at rest or after stimulation $(n=8,13,7,7) . \boldsymbol{F}$, Quantification of Cttn fluorescence intensity at muscle 4 synapses in control and para $a^{\text {ts }}$ animals raised at $29^{\circ} \mathrm{C}$, at rest or after stimulation $(n=12,11,12,11) . \mathbf{G}$, Quantification of $\mathrm{Cttn}$ fluorescence intensity at synapses in muscle 4 in control and syt $1^{N 13} /$ syt $^{A D 4}$ animals, at rest or after stimulation $(n=10,13,11,16)$. n.s., Not significant. ${ }^{* *} p<0.01 ;{ }^{* * *} p<0.001$; ${ }^{* * * *} p<0.0001$ (ANOVA with post hoc Dunnett and Tukey tests). Data represent mean \pm SEM. Scale bar: $10 \mu \mathrm{m}$.

the emergence of filopodia in chick embryonic sensory neurons (Spillane et al., 2012), we think it likely that Cttn has a more general role in regulating membrane dynamics during plastic events in both vertebrates and invertebrates.

\section{The increase of synaptic Cortactin during repeated} stimulation is dependent on activity

We then asked whether blocking activity during repeated stimulation could block the increase of synaptic Cortactin. To do so, we first examined para $^{\text {tsl }}$ mutants. para encodes the $\alpha$ subunit of the voltage-gated sodium channel required for the generation of sodium-dependent action potentials (Ganetzky, 1984; Littleton and Ganetzky, 2000). Because para $^{\text {tsl }}$ is a thermosensitive mutant whose function is perturbed at restrictive temperatures, we incubated the animals (control and $\operatorname{para}^{\text {ts }}$ ) at $29^{\circ} \mathrm{C}$ for $4 \mathrm{~h}$ before performing repeated stimulation. We found that the number of ghost boutons in the para ${ }^{\text {ts } 1}$ animals was significantly decreased (controls, $8.7 \pm 1.3, n=20$; para $^{\text {tsl }}$, $1.9 \pm 0.7, n=14$ ), as reported previously (Ataman et al., 2008). In addition, we found that unstimulated para ${ }^{t s l}$ animals had the same amount of synaptic Cttn as unstimulated controls $(p=0.9)$ and that, upon stimulation, control animals showed an increase of $151 \%(p<0.0001)$ in Cttn staining intensity. In contrast, the amount of synaptic Cttn in stimulated para ${ }^{\text {tsl }}$ animals only increased by $65 \%$ and was not significantly different from unstimulated controls $(p=0.09)$ or unstimulated para $a^{t s I}$ animals $(p=0.1)$, while being significantly reduced $(p=0.04)$ compared to stimulated controls (Fig. $4 F$ ).

To confirm that activity is necessary for synaptic $\mathrm{Cttn}$ increase, and because high $\mathrm{K}^{+}$ depolarization can potentially provoke neurotransmitter release in the absence of action potentials, we also examined animals with perturbed neurotransmitter release (Fig. 4G). Syt1 mutants $\left(s y t 1^{N 13} / s y t 1^{A D 4}\right.$ ) showed significant reduction in synaptic plasticity after stimulation (controls, $8.25 \pm$ $1.5, n=8 ;$ syt 1 mutants, $3.8 \pm 1.2, n=10$ ) in accordance with previous publications (Piccioli and Littleton, 2014). We then looked at the abundance of synaptic $\mathrm{Cttn}$ in these different animals. First, we established that at rest there was no difference between controls and syt1 animals (Fig. $4 G ; p=$ $0.28)$. We then asked whether stimulation provoked a change in the abundance of synaptic Cttn. In controls we observed a significant increase in Cttn staining intensity $(p<$ 0.0001 ), while there was no increase in syt 1 mutant animals $(p=0.77)$. This series of data shows that the increase of Cttn at the synapse depends on action potentials and neurotransmitter release. We conclude that sustained neuronal activity is required for the increase of synaptic Cortactin.

The increase of synaptic Cortactin during repeated stimulation is dependent on $\mathrm{Wg}$ expression

Since repeated stimulation provokes an increase in synaptic $\mathrm{Wg}$ expression that is necessary for activity-dependent modification in synaptic structure (Ataman et al., 2008), we asked whether Wg was also necessary for the increase in Cttn. Because Wg is necessary for embryonic development and for NMJ differentiation (Packard et al., 2002), we used a thermosensitive wg mutant $\left(w g^{t s}\right)$ that shows wild-type function at $18^{\circ} \mathrm{C}$ and behaves like a strong loss of function mutation at $30^{\circ} \mathrm{C}$ (van den Heuvel et al., 


\section{A Unstimulated control}
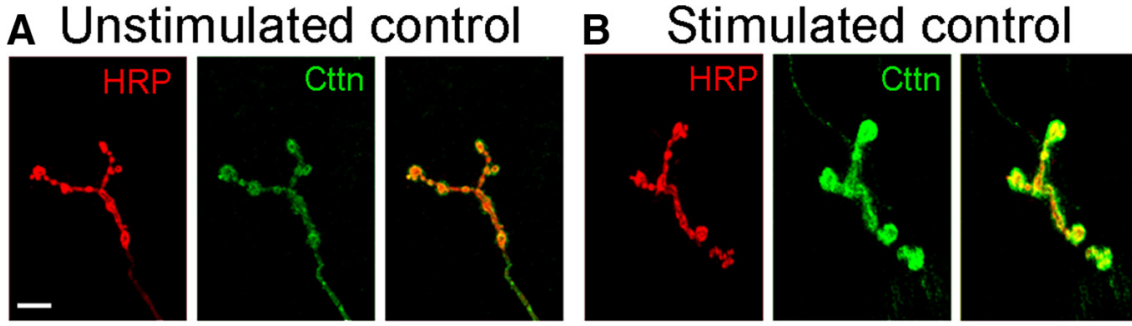

c Unstimulated $\mathrm{Wg}^{\mathrm{ts}}$
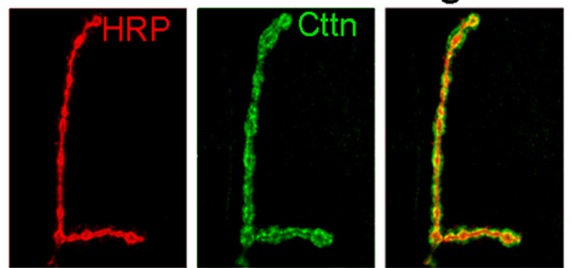

D Stimulated $\mathrm{Wg}^{\mathrm{ts}}$
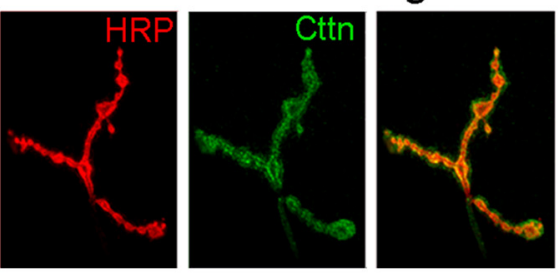

E

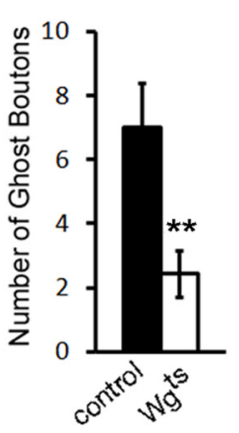

$\mathbf{F}$
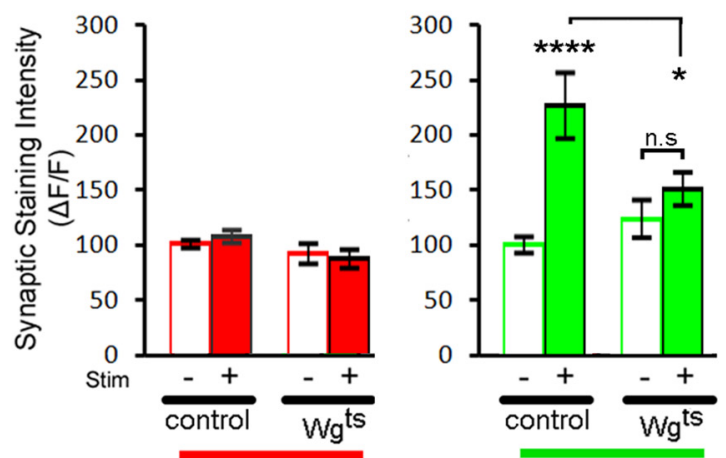

G

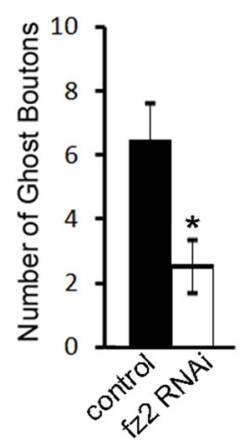

H

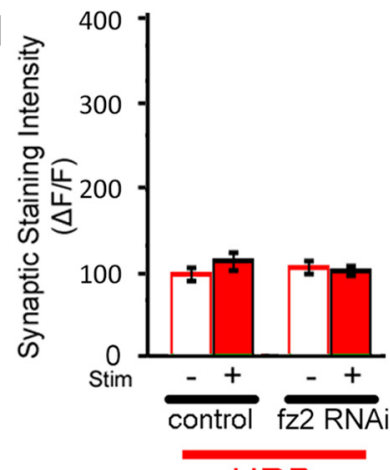

HRP

HRP

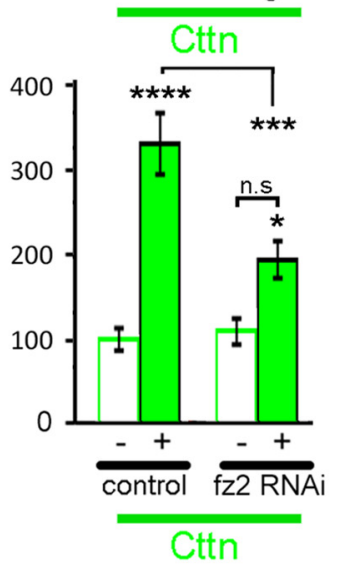

J

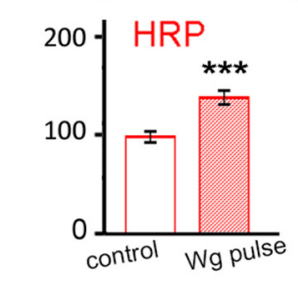

1993). In these experiments, animals (control and $w g^{t s}$ ) were left to develop at $18^{\circ} \mathrm{C}$ and shifted to $30^{\circ} \mathrm{C}$ for $5 \mathrm{~h}$ before application of the repeated stimulation protocol at $30^{\circ} \mathrm{C}$. We then determined the number of ghost boutons and the quantity of synaptic Cttn immunostaining (Fig. 5). We first showed that under these conditions, repeated stimulation was able to provoke the formation of ghost boutons $(7 \pm 1.4)$ at control synapses, while $w g^{t s}$ animals showed a strong decrease in ghost boutons $(2.4 \pm 0.7)$ after repeated stimulation (Fig. $5 E$ ). We then asked whether the increase in Cttn at stimulated synapses was dependent on Wg expression. Control preparations showed increased synaptic Cttn levels after repeated stimulation (a 125\% increase), while Wg-deficient synapses showed no significant change (Fig. $5 A-$ $D, F)$. Interestingly, there was no difference in Cttn expression when we compared levels of synaptic fluorescence in unstimulated controls (100 $\pm 7.4 \%$, $n=15)$ to unstimulated $w g^{t s}$ synapses $(124 \pm 16.8 \%, n=15$; Fig. $5 A, C, F)$. This shows that basal levels of synaptic Cttn are not affected by acute loss of Wg function, but that Wg signaling is required for the increase of synaptic Cttn provoked by repeated stimulation.

To confirm this result, we targeted the presynaptic Wg receptor by expressing an RNAi transgene against frizzled 2 ( $f z 2$ ) in neurons (D42-Gal4/UAS-fz2 RNAi). We found that these transgenic animals showed decreased plasticity after repeated stimulation (Fig. 5G). We then asked whether Cttn expression was affected (Fig. $5 H)$. There was no difference in the level of Cttn intensity at rest between control (D42Gal4/+) and the fz2 RNAi knockdown animals. Upon stimulation, both control and the $f z 2$ RNAi knockdown animals showed significant increases in Cttn fluorescence intensity $(232 \%, p<0.0001$ and $79 \%, p=$ 0.026 respectively). However, the magnitude of the increase between the two genotypes was significantly different $(p=$ 0.0004; Fig. 5H), showing that a reduction

muscle 4 synapses in control synapses (elav ${ }^{\text {C155 }}$-Gal4/+) and synapses overexpressing Wg (elav ${ }^{155}$-Gal4/+; UAS-Wg/+). $n=13,11$. J, Quantification of HRP and Cttn fluorescence intensity at muscle 4 synapses in control synapses (elav ${ }^{\mathrm{C} 155}$. $\mathrm{Ga} 14 /+$ ) and synapses overexpressing Wg (elav ${ }^{\mathrm{C} 155}$-Gal4/+; Tub-Gal80 $/ 5 / \mathrm{UAS}-\mathrm{Wg}$ ) after a $2 \mathrm{~h}$ pulse at $29^{\circ} \mathrm{C} . n=10$, 18. n.s., Not significant. ${ }^{*} p<0.05 ;{ }^{* *} p<0.01 ;{ }^{* * *} p<$ $0.001 ;{ }^{* * * *} p<0.0001$ [ANOVA with post hoc Dunnett and Tukey tests $(\boldsymbol{F}, \boldsymbol{H})$ or $t$ test $(\boldsymbol{E}, \boldsymbol{G}, \boldsymbol{I}, \boldsymbol{J})]$. Data represent mean \pm SEM. Scale bars: $10 \mu \mathrm{m}$.
Figure 5. The increase in synaptic Cortactin after repeated stimulation is dependent on Wg expression. $\boldsymbol{A}-\boldsymbol{D}$, Unstimulated ( $\boldsymbol{A})$ and stimulated $(\boldsymbol{B})$ control synapses and unstimulated $(\boldsymbol{C})$ and stimulated $(\boldsymbol{D}) \mathrm{wg}$ ts synapses at $30^{\circ} \mathrm{C}$. $\boldsymbol{E}$, Quantification of the number of ghost boutons induced by repeated stimulations at $30^{\circ} \mathrm{C}$ in control and $w g^{t s}$ synapses $(n=14,7)$. $\boldsymbol{F}$, Quantification of $\operatorname{HRP}(n=15,16,15,14)$ and $\mathrm{Cttn}(n=15,16,15,21)$ fluorescence intensity at muscle 4 synapses in control and $w g^{t s}$ synapses at $30^{\circ} \mathrm{C}$, at rest or after stimulation. $G$, Quantification of the number of ghost boutons induced by repeated stimulations in contro (D42-gal4/+; $n=10)$ synapses and synapses expressing RNAi against Fz2 in motoneurons (D42-gal4/UAS Fz2 RNAi; $n=10$ ). $\boldsymbol{H}$, Quantification of $\operatorname{HRP}(n=17,17,16,19)$ and $\operatorname{ttn}(n=13,13,12,19)$ fluorescence intensity at muscle 4 synapses in contro synapses and synapses expressing RNAi against Fz2 in motoneurons. I, Quantification of HRP and Cttn fluorescence intensity a 

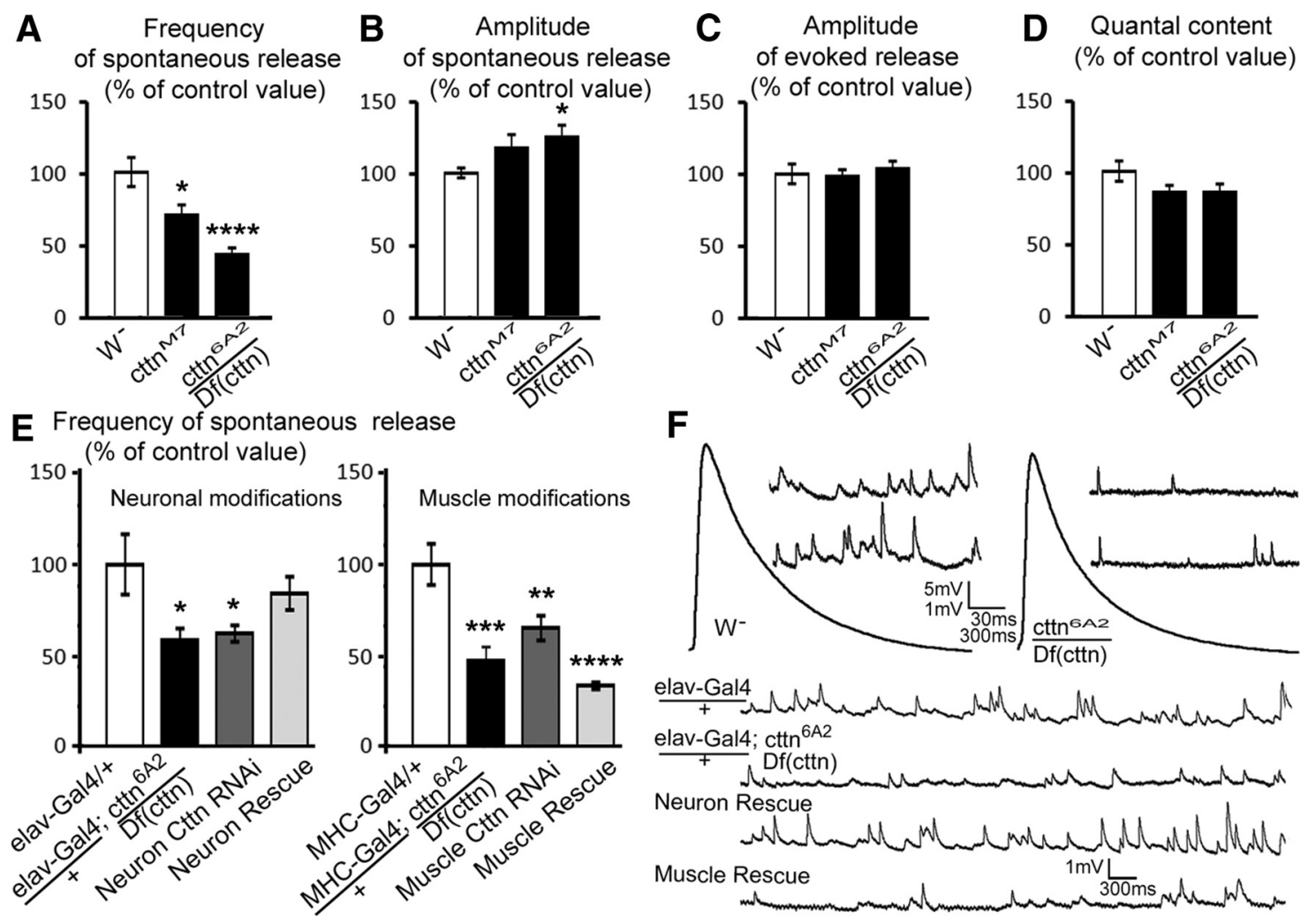

Figure 6. Presynaptic Cortactin regulates spontaneous release frequency. Quantification of the frequency of spontaneous release $(n=25,13,15 ; A)$, spontaneous release amplitude $(n=25,13$, $15 ; \boldsymbol{B})$, evoked release amplitude $(n=12,12,15 ; C)$, and quantal content $(n=12,12,15 ; \boldsymbol{D})$ in $w^{-}$, $c t t n^{M 7}$, and $c t t^{6 A 2} / D f(c t n)$ animals. $E$, Quantification of the frequency of spontaneous release in animals with a genotype affecting neurons: neuronal driver in a control background (elav-Gal4/+) or cttn mutant background [elav-Gal4/+; +; $\left.\mathrm{cttn}{ }^{6 \mathrm{A2}} / \mathrm{Df}(\mathrm{cttn})\right]$, neuron cttn RNAi (elavGal4/+; UAS-cttn $\left.{ }^{\text {RAAi }}\right)$, and neuron rescue [elav-Gal4/+; UAS-cttn/+; $\mathrm{Cttn}^{6 A 2} / \mathrm{Df}(\mathrm{cttn}) ; n=15,10,14,12$ ]. Quantification of the frequency of spontaneous release in animals with a genotype

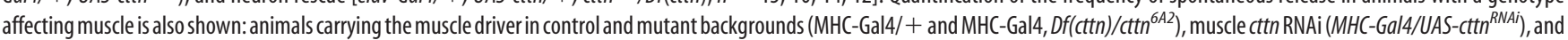
muscle rescue [UAS-cttn/+; MHC-Gal4, Df(cttn)/cttn $\left.{ }^{6 A 2}\right] . n=7,8,8,7$. F, Representative spontaneous and evoked traces from control, mutant, and rescue animals. ${ }^{*} p<0.05$; ** $p<0.01$; ${ }^{* * *} p<0.001 ;{ }^{* * * *} p<0.0001$ (ANOVA with post hoc Dunnett test). Data represent mean \pm SEM.

in presynaptic Fz2 expression is sufficient to hinder the increase in synaptic Cttn after stimulation.

Because $\mathrm{Wg}$ is required for the increase of Cttn during activity-dependent synaptic plasticity, we wondered whether Wg expression was sufficient to induce an increase in Cttn levels. To test this, we first overexpressed wg in neurons ( elav $^{\mathrm{Cl} 55}$-Gal4/+; $U A S-w g /+)$ and asked whether we could detect a difference in Cttn intensity when compared to control (Fig. 5I). There were no notable differences in expression. Because these animals overexpressed wg since embryogenesis (elav ${ }^{\mathrm{Cl} 5}$-Gal4 is a postmitotic pan-neuronal driver), we hypothesized that any potential changes in Cttn expression might not have lasted until the stage at which we are examining the NMJ. We then examined transgenic animals containing a thermosensitive inhibitor of the Gal4/UAS system, Gal80 ${ }^{t s}$ (McGuire et al., 2004), under the control of the ubiquitous tubulin (Tub) promoter in addition to elav ${ }^{C 155}$. Gal4/+; UAS-wg/+. These animals do not express an excess of $\mathrm{Wg}$ at the permissive temperature $\left(20^{\circ} \mathrm{C}\right)$, and $\mathrm{Wg}$ overexpression is controlled by the precise time at which the animals are shifted to a restrictive temperature $\left(29^{\circ} \mathrm{C}\right)$. We therefore decided to shift these animals to $29^{\circ} \mathrm{C} 2 \mathrm{~h}$ before fixation and quantification of the intensity of synaptic proteins to mimic a pulse of $\mathrm{Wg}$ overexpression. Under these conditions, we noticed a significant increase of synaptic Cttn (58\%) and HRP staining intensity (40\%) when compared to control animals submitted to the same treatment. This result suggests that an acute increase of $\mathrm{Wg}$ is sufficient to modify the abundance of Cttn and other presynaptic proteins.

\section{Presynaptic Cortactin regulates spontaneous} release frequency

Because Cttn is present presynaptically and postsynaptically at the NMJ and because actin regulation has been linked to several aspects of synapse assembly (Nelson et al., 2013), we turned to the larval NMJ preparation to analyze the role of Cttn on synaptic physiology. We first noticed a striking difference in the frequency of mEPSPs in cttn mutants compared to control. In both mutant conditions, the frequency of spontaneous release was greatly reduced. The spontaneous release frequency in $\mathrm{cttn}^{M 7}$ homozygous animals was $71 \%$ of the control value, while it was $44 \%$ of control in $c t n^{6 A 2} / D f(c t t n)$ (Fig. 6A). We then investigated whether this deficit in spontaneous release frequency was due to the lack of presynaptic or postsynaptic Cttn. To do so, we first recorded from the $c t t n^{6 A 2} / D f(c t t n)$ mutants in genetic backgrounds containing a neuron or a muscle driver. In these backgrounds, the frequency of mEPSPs was reduced to 59 and $49 \%$ of control values (Fig. 6E,F). Using these backgrounds, we were able to express the full cttn cDNA presynaptically (neuron rescue) or postsynaptically (muscle rescue). We found that animals expressing Cttn only in neurons had a frequency of spontaneous release similar to control flies ( $84 \pm 9 \%$; $p=0.62$; Fig. $6 E, F)$. On the contrary, animals expressing Cttn in muscles only did not show rescue toward the control phenotype; their frequency of spontaneous release was $34 \pm 2 \%$ of the control value $(p<0.0001$; Fig. $6 E, F)$. In addition, we used RNAi transgenes to drive the knockdown of Cttn in neurons or muscles. When we drove $c t t n$ RNAi in neurons, the frequency of spontaneous release was reduced to 
$62 \pm 4 \%$ compared to control preparations (Fig. 6E). This result, along with the neuronal rescue, suggests that presynaptic Cttn is critical to the rate of spontaneous synaptic vesicle fusion at the Drosophila NMJ. However, we found that when we drove $c t t n$ RNAi expression in muscles, the frequency of spontaneous release $(65 \pm 7 \%)$ was also significantly different from control (Fig. 6E). Hence, even though postsynaptic Cttn rescue does not restore mini frequency, we cannot discard the possibility that postsynaptic Cttn could somehow regulate mini frequency.

We also noticed a modest increase in mEPSP amplitude in one of the two mutant strains we studied (Fig. 6B). Indeed, the $\mathrm{cttn}^{6 A 2} / D f(\mathrm{cttn})$ animals had a mean mEPSP amplitude that was $125 \pm 8 \%$ of the control value $(p=0.01)$. However, there was no significant increase in mEPSP amplitude in the $c t t n^{M 7}$ mutants $(p=0.1)$. In addition, our attempts to rescue the $c t t n^{6 A 2} / D f(c t t n)$ mEPSP amplitude phenotype by driving Cttn presynaptically or postsynaptically were not successful (data not shown). Because this phenotype was subtle, not consistently observed in different mutant conditions, and could not be rescued by presynaptic or postsynaptic expression, we concluded that it is most likely due to a synthetic genetic interaction between $\mathrm{cttn}$ and one of the genes affected in the $D f(c t t n)$ deficiency. Unlike the spontaneous release frequency, this mEPSP amplitude phenotype cannot therefore be attributed directly to Cttn. We did not observe any differences in EPSP amplitude or quantal content (Fig. $6 C, D$ ), suggesting that $\mathrm{Cttn}$ does not affect evoked synaptic release.

Presynaptic Cortactin is necessary for the rapid activitydependent potentiation of spontaneous release frequency We then turned to the ability of the synapse to be plastic in the absence of Cttn. Another characteristic of rapid activitydependent plasticity at the NMJ is the increase in the frequency of spontaneous release events, as detected by mEPSPs (Ataman et al., 2008). It is thought to reflect a change in the intrinsic properties of the synapse and/or the "unsilencing" of active zones as seen in mammalian models (Yao et al., 2006). It is important to note that the increase in mEPSP frequency is independent of the activity-induced changes in synaptic morphology. Indeed, since the postsynaptic differentiation of the de novo ghost boutons occurs long after we measure mEPSP frequency, the ghost boutons cannot be responsible for the increase in spontaneous release frequency. Electrophysiological changes are therefore not a consequence of morphological modifications. Consistent with previously published data, we find that $w^{-}$control preparations show a potentiation of spontaneous release: nonstimulated synapses show a mEPSP frequency of $2.46 \mathrm{~Hz}$, while stimulated synapses show a mEPSP frequency of $5.1 \mathrm{~Hz}$ (an increase of 107\%;
Fig. $7 A, E)$. We quantified this data and presented it as a frequency potentiation index (Ataman et al., 2008; a ratio of frequency of spontaneous release between stimulated and unstimulated NMJs from a given genotype). The $w^{-}$control preparations showed a potentiation index of 2.07 (Fig. 7E), while elav-Gal4 and MHC-Gal4 controls showed a potentiation indices of 1.69 (Fig. $7 F$ ) and $1.51(G)$. Since Cttn is critical for the structural changes associated with rapid activity-dependent synaptic plasticity, we wanted to test whether it was also essential for the potentiation of spontaneous release. We found that there was no activity-induced potentiation of mEPSP frequency in $\mathrm{cttn}^{6 A 2} / \mathrm{Df}$ $(\mathrm{cttn})$ and $\mathrm{cttn}^{M 7}$ mutant animals (potentiation indices of 0.9 and 1.03; Fig. $7 B, E)$. Hence, we conclude that $\mathrm{Cttn}$ is necessary for this process at the NMJ. We then asked whether Cttn was required presynaptically or postsynaptically. Flies expressing $\mathrm{cttn}$ RNAi in neurons also showed no potentiation of spontaneous release frequency (potentiation index of 0.67 ; Fig. $7 C, F$ ). In contrast, when $c t t n$ RNAi was expressed in the muscle, there was still a significant increase in the frequency of spontaneous release after stimulation (potentiation index of $1.54 ; p=0.008$; Fig. $7 D, G)$ and no significant difference compared to stimulated 
MHC-Gal4 controls ( $p=0.99$ ). In addition, we performed rescue experiments where we expressed cttn cDNA exclusively in neurons or in muscles of otherwise cttn null mutant animals. We find that Cttn neuronal expression is sufficient to restore potentiation of spontaneous release frequency (index of 1.48; Fig $7 F$ ), while expression of muscle $\mathrm{Cttn}$ does not rescue the phenotype (index of 1.13; Fig 7G). We conclude that it is the presynaptic Cttn that is essential for the rapid activity-dependent potentiation of spontaneous release frequency. It is therefore likely that these changes in spontaneous release are a consequence of a Cttndependent modification of presynaptic release sites.

\section{Discussion}

Major signaling molecules, such as Netrin, $\operatorname{TNF} \alpha, \operatorname{TGF} \beta$, and Wnt, are essential for the plasticity of the nervous system (Poon et al., 2013), and molecules able to modulate cytoskeleton organization are likely intracellular effectors of these signals. For example, Wnt signaling has been associated with the ability to modify microtubule stability. Indeed, the downstream kinase Sgg/Gsk $3 \beta$ can phosphorylate microtubule-associated proteins and affect microtubule stability, which in turn affects synapse growth and stability (Goold et al., 1999; Packard et al., 2002; Ciani et al., 2004; Miech et al., 2008). Previously, the actin regulator Cofilin was shown to be essential for the morphological changes associated with repeated stimulation, but it is not clear whether its phosphorylation state or abundance is part of a switch that transduces activity-dependent synaptic growth (Piccioli and Littleton, 2014).

Our present work shows that $\mathrm{Wg} / \mathrm{Wnt}$, the expression of which increases considerably during repeated stimulation (Ataman et al., 2008), is required for the acute increase of Cortactin, a major membrane protrusion regulator that we show here is of great importance for activity-dependent synaptic plasticity. We argue that the dynamic, activity-dependent synaptic expression of Cortactin determines the degree of plasticity. Interestingly, Cortactin synaptic levels can more than double in $90 \mathrm{~min}$, while its presence at the synapse appears quite unstable, allowing for the modulation of its expression over a fairly short timescale. In any case, it is important to note that these results could be explained by invoking a direct increase in the transcription of Cttn during stimulation or by the stabilization of synaptic Cttn by proteins requiring de novo transcription. Similarly, in chick embryonic sensory neurons, Cortactin has been shown to respond to NGF application, which provokes a rapid translation-dependent increase of axonal Cortactin, leading in turn to collateral branching and the emergence of filopodia (Spillane et al., 2012).

In addition, our work defines a novel presynaptic role for Cortactin in regulating activity-dependent synaptic plasticity. Previously, Cortactin's known role in synaptic plasticity has been restricted to the postsynaptic cell only. For example, it is known to be involved in activity-dependent spine morphogenesis, where it is redistributed in response to synaptic stimulation and NMDA receptor activation (Hering and Sheng, 2003; Iki et al., 2005; Chen and Hsueh, 2012; Lin et al., 2013). Recently, interactions between Shank and Cortactin have been proposed to regulate actin dynamics underlying dendritic spine morphology and function (MacGillavry et al., 2016). Our data suggest a more general role for Cortactin in regulating membrane/actin dynamics during plastic events on both sides of the synapse. In particular, we have shown that presynaptic Cortactin is necessary for the potentiation of spontaneous release, suggesting that it can modify the structure and/or function of active zones. The mechanisms by which the increase in spontaneous release is achieved after stimulation remain unclear. The increase in spontaneous release fre- quency could be explained by the recruitment of new active zones. While we cannot discard this possibility, we can be sure that it is not a consequence of ghost bouton formation since, at the stage of our electrophysiological recordings, they are devoid of postsynaptic differentiation. Another possible mechanism is the "unsilencing" of existing active zones, a phenomenon described before in cultured hippocampal neurons and shown to be actin and activity-dependent (Yao et al., 2006). It could also be due to changes in the intrinsic properties or structure of the presynapse. For example, it could somehow antagonize the actions of molecules such as Complexin, which downregulates the frequency of spontaneous vesicle release and was previously linked to activity-dependent synaptic plasticity (Huntwork and Littleton, 2007; Jorquera et al., 2012; Wragg et al., 2013; Cho et al., 2015). Interestingly, within a mutant background, overexpression of Cortactin can increase actin polymerization and rescue synaptic vesicle clustering (Sun and Bamji, 2011), suggesting that Cortactin is able to modulate some aspects of the presynaptic structure. A challenge for the future will be to determine which mechanisms require Cttn expression and are essential for activity-dependent synaptic plasticity.

Our work shows that the increase of synaptic Cttn depends on activity and Wg signaling. Because it has been shown that neuronal activity leads to an increase of Wg at the synapse (Ataman et al., 2008), we hypothesize that activity induces increased synaptic $\mathrm{Wg}$ that, in turn, induces increased synaptic Cttn. Even though we cannot rule out that Wg signaling might occur at the level of the cell body, it is tempting to imagine a regulatory Wg transduction pathway at the synapse. Indeed, Wg signaling can be transduced through different signaling pathways (Koles and Budnik, 2012), and most of them have been shown to be present at the NMJ. The canonical Wg pathway has been characterized at the NMJ where the protein kinase Sgg/Gsk3 $\beta$ is involved in regulating activity dependent synaptic plasticity (Ataman et al., 2008). Because $\mathrm{Sgg} / \mathrm{Gsk} 3 \beta$ controls the transcription factor $\mathrm{Arm} / \beta$ catenin and because activity-dependent synaptic plasticity depends on de novo transcription (Ataman et al., 2008; present study), one could envision that $\mathrm{Sgg} / \mathrm{Gsk} 3 \beta$ and $\mathrm{Arm} / \beta$-catenin are responsible for Cttn's increase. Nevertheless, Sgg/Gsk $3 \beta$ also has a synaptic role independent of Arm/ $\beta$-catenin, in controlling microtubule structure (Goold et al., 1999; Packard et al., 2002; Ciani et al., 2004; Miech et al., 2008). One possibility is that the increase of synaptic Cttn is due to constant transcription and local stabilization under the control of Sgg/Gsk3 $\beta$.

Another Wg pathway, the noncanonical $\mathrm{Ca}^{2+}$ pathway, the output of which is transcriptional regulation through nuclear factor of activated T-cells, has also been shown to regulate growth and plasticity at the synapse (Freeman et al., 2011). It could thus be part of the regulation of Cttn during activity-dependent plasticity. The other noncanonical pathway, the planar cell polarity pathway, has not been characterized at the NMJ yet, but it is interesting to note that the activity of the Jun kinase, a key element of this pathway, has been linked to a notable increase in Cttn transcription in Drosophila embryos (Jasper et al., 2001). Deciphering how these different Wg pathways lead to an increase in synaptic Cttn will be an exciting challenge for the future.

In any case, we present a novel link between a major plasticity signaling molecule, $\mathrm{Wg} / \mathrm{Wnt}$, and a known actin modifier, Cortactin. Interestingly, Cortactin is overexpressed in many cancers and is a marker for aggressive tumors (MacGrath and Koleske, 2012). The role of Cortactin in synaptic plasticity that we describe here is reminiscent of its function in promoting cancer, where its role in cancer cell invasion and metastasis is linked to the ability 
to control actin-driven protrusions (MacGrath and Koleske, 2012). Our finding indicates that Cortactin is downstream of Wnt signaling, also involved in numerous cancers (Anastas and Moon, 2013). It would be of great interest to determine whether the subset of cancers that involve an increase in Wnt signaling also exhibit increases in Cortactin levels.

\section{References}

Ammer AG, Weed SA (2008) Cortactin branches out: roles in regulating protrusive actin dynamics. Cell Motil Cytoskeleton 65:687-707. CrossRef Medline

Anastas JN, Moon RT (2013) WNT signalling pathways as therapeutic targets in cancer. Nat Rev Cancer 13:11-26. Medline

Ataman B, Ashley J, Gorczyca M, Ramachandran P, Fouquet W, Sigrist SJ, Budnik V (2008) Rapid activity-dependent modifications in synaptic structure and function require bidirectional Wnt signaling. Neuron 57: 705-718. CrossRef Medline

Bhambhvani HP, Simmons M, Haroutunian V, Meador-Woodruff JH (2016) Decreased expression of cortactin in the schizophrenia brain. Neuroreport 27:145-150. CrossRef Medline

Bosch M, Hayashi Y (2012) Structural plasticity of dendritic spines. Curr Opin Neurobiol 22:383-388. CrossRef Medline

Brand AH, Perrimon N (1993) Targeted gene expression as a means of altering cell fates and generating dominant phenotypes. Development 118: 401-415. Medline

Budnik V, Koh YH, Guan B, Hartmann B, Hough C, Woods D, Gorczyca M (1996) Regulation of synapse structure and function by the Drosophila tumor suppressor gene dlg. Neuron 17:627-640. CrossRef Medline

Chen YK, Hsueh YP (2012) Cortactin-binding protein 2 modulates the mobility of cortactin and regulates dendritic spine formation and maintenance. J Neurosci 32:1043-1055. CrossRef Medline

Cho RW, Buhl LK, Volfson D, Tran A, Li F, Akbergenova Y, Littleton JT (2015) Phosphorylation of complexin by PKA regulates activitydependent spontaneous neurotransmitter release and structural synaptic plasticity. Neuron 88:749-761. CrossRef Medline

Ciani L, Krylova O, Smalley MJ, Dale TC, Salinas PC (2004) A divergent canonical WNT-signaling pathway regulates microtubule dynamics: dishevelled signals locally to stabilize microtubules. J Cell Biol 164:243-253. CrossRef Medline

Cohen SM, Li B, Tsien RW, Ma H (2015) Evolutionary and functional perspectives on signaling from neuronal surface to nucleus. Biochem Biophys Res Commun 460:88-99. CrossRef Medline

Crabtree GW, Gogos JA (2014) Synaptic plasticity, neural circuits, and the emerging role of altered short-term information processing in schizophrenia. Front Synaptic Neurosci 6:28. Medline

DiAntonio A, Schwarz TL (1994) The effect on synaptic physiology of synaptotagmin mutations in Drosophila. Neuron 12:909-920. CrossRef Medline

Freeman A, Franciscovich A, Bowers M, Sandstrom DJ, Sanyal S (2011) NFAT regulates pre-synaptic development and activity-dependent plasticity in Drosophila. Mol Cell Neurosci 46:535-547. CrossRef Medline

Ganetzky B (1984) Genetic studies of membrane excitability in Drosophila: lethal interaction between two temperature-sensitive paralytic mutations. Genetics 108:897-911. Medline

Goley ED, Welch MD (2006) The ARP2/3 complex: an actin nucleator comes of age. Nat Rev Mol Cell Biol 7:713-726. CrossRef Medline

Goold RG, Owen R, Gordon-Weeks PR (1999) Glycogen synthase kinase 3 beta phosphorylation of microtubule-associated protein $1 \mathrm{~B}$ regulates the stability of microtubules in growth cones. J Cell Sci 112:3373-3384. Medline

Hell JW (2014) CaMKII: claiming center stage in postsynaptic function and organization. Neuron 81:249-265. CrossRef Medline

Hering H, Sheng M (2003) Activity-dependent redistribution and essential role of cortactin in dendritic spine morphogenesis. J Neurosci 23:1175911769. Medline

Horn KE, Glasgow SD, Gobert D, Bull SJ, Luk T, Girgis J, Tremblay ME, McEachern D, Bouchard JF, Haber M, Hamel E, Krimpenfort P, Murai KK, Berns A, Doucet G, Chapman CA, Ruthazer ES, Kennedy TE (2013) DCC expression by neurons regulates synaptic plasticity in the adult brain. Cell Rep 3:173-185. CrossRef Medline

Huntwork S, Littleton JT (2007) A complexin fusion clamp regulates spon- taneous neurotransmitter release and synaptic growth. Nat Neurosci 10: 1235-1237. CrossRef Medline

Iki J, Inoue A, Bito H, Okabe S (2005) Bi-directional regulation of postsynaptic cortactin distribution by BDNF and NMDA receptor activity. Eur J Neurosci 22:2985-2994. CrossRef Medline

Jan LY, Jan YN (1982) Antibodies to horseradish peroxidase as specific neuronal markers in Drosophila and in grasshopper embryos. Proc Natl Acad Sci U S A 79:2700-2704. CrossRef Medline

Jasper H, Benes V, Schwager C, Sauer S, Clauder-Münster S, Ansorge W, Bohmann D (2001) The genomic response of the Drosophila embryo to JNK signaling. Dev Cell 1:579-586. CrossRef Medline

Jorquera RA, Huntwork-Rodriguez S, Akbergenova Y, Cho RW, Littleton JT (2012) Complexin controls spontaneous and evoked neurotransmitter release by regulating the timing and properties of synaptotagmin activity. J Neurosci 32:18234-18245. CrossRef Medline

Katsube T, Takahisa M, Ueda R, Hashimoto N, Kobayashi M, Togashi S (1998) Cortactin associates with the cell-cell junction protein ZO-1 in both Drosophila and mouse. J Biol Chem 273:29672-29677. CrossRef Medline

Katz F, Moats W, Jan YN (1988) A carbohydrate epitope expressed uniquely on the cell surface of Drosophila neurons is altered in the mutant nac (neurally altered carbohydrate). EMBO J 7:3471-3477. Medline

Kirkbride KC, Sung BH, Sinha S, Weaver AM (2011) Cortactin: a multifunctional regulator of cellular invasiveness. Cell Adh Migr 5:187-198. CrossRef Medline

Koles K, Budnik V (2012) Wnt signaling in neuromuscular junction development. Cold Spring Harb Perspect Biol 4:a008045. Medline

Lin YC, Yeckel MF, Koleske AJ (2013) Abl2/Arg controls dendritic spine and dendrite arbor stability via distinct cytoskeletal control pathways. J Neurosci 33:1846-1857. CrossRef Medline

Littleton JT, Ganetzky B (2000) Ion channels and synaptic organization: analysis of the Drosophila genome. Neuron 26:35-43. CrossRef Medline

Littleton JT, Stern M, Perin M, Bellen HJ (1994) Calcium dependence of neurotransmitter release and rate of spontaneous vesicle fusions are altered in Drosophila synaptotagmin mutants. Proc Natl Acad Sci U S A 91:10888-10892. CrossRef Medline

MacGillavry HD, Kerr JM, Kassner J, Frost NA, Blanpied TA (2016) Shankcortactin interactions control actin dynamics to maintain flexibility of neuronal spines and synapses. Eur J Neurosci 43:179-193. CrossRef Medline

MacGrath SM, Koleske AJ (2012) Cortactin in cell migration and cancer at a glance. J Cell Sci 125:1621-1626. CrossRef Medline

Maldonado C, Alicea D, Gonzalez M, Bykhovskaia M, Marie B (2013) Adar is essential for optimal presynaptic function. Mol Cell Neurosci 52:173180. Medline

Marie B, Sweeney ST, Poskanzer KE, Roos J, Kelly RB, Davis GW (2004) Dap160/intersectin scaffolds the periactive zone to achieve high-fidelity endocytosis and normal synaptic growth. Neuron 43:207-219. CrossRef Medline

Marie B, Pym E, Bergquist S, Davis GW (2010) Synaptic homeostasis is consolidated by the cell fate gene gooseberry, a Drosophila pax3/7 homolog. J Neurosci 30:8071-8082. CrossRef Medline

McGuire SE, Mao Z, Davis RL (2004) Spatiotemporal gene expression targeting with the TARGET and gene-switch systems in Drosophila. Sci STKE 2004:pl6. Medline

Miech C, Pauer HU, He X, Schwarz TL (2008) Presynaptic local signaling by a canonical wingless pathway regulates development of the Drosophila neuromuscular junction. J Neurosci 28:10875-10884. CrossRef Medline

Nelson JC, Stavoe AK, Colón-Ramos DA (2013) The actin cytoskeleton in presynaptic assembly. Cell Adh Migr 7:379-387. CrossRef Medline

Packard M, Koo ES, Gorczyca M, Sharpe J, Cumberledge S, Budnik V (2002) The Drosophila Wnt, wingless, provides an essential signal for pre- and postsynaptic differentiation. Cell 111:319-330. CrossRef Medline

Piccioli ZD, Littleton JT (2014) Retrograde BMP signaling modulates rapid activity-dependent synaptic growth via presynaptic LIM kinase regulation of Cofilin. J Neurosci 34:4371-4381. CrossRef Medline

Poon VY, Choi S, Park M (2013) Growth factors in synaptic function. Front Synaptic Neurosci 5:6. Medline

Rosso SB, Sussman D, Wynshaw-Boris A, Salinas PC (2005) Wnt signaling through Dishevelled, Rac and JNK regulates dendritic development. Nat Neurosci 8:34-42. CrossRef Medline

Salinas PC (2012) Wnt signaling in the vertebrate central nervous system: 
from axon guidance to synaptic function. Cold Spring Harb Perspect Biol 4:a008003. Medline

Snow PM, Patel NH, Harrelson AL, Goodman CS (1987) Neural-specific carbohydrate moiety shared by many surface glycoproteins in Drosophila and grasshopper embryos. J Neurosci 7:4137-4144. Medline

Somogyi K, Rørth P (2004) Cortactin modulates cell migration and ring canal morphogenesis during Drosophila oogenesis. Mech Dev 121:57-64. CrossRef Medline

Spillane M, Ketschek A, Donnelly CJ, Pacheco A, Twiss JL, Gallo G (2012) Nerve growth factor-induced formation of axonal filopodia and collateral branches involves the intra-axonal synthesis of regulators of the actin-nucleating Arp2/3 complex. J Neurosci 32:17671-17689. CrossRef Medline

Stricker J, Falzone T, Gardel ML (2010) Mechanics of the F-actin cytoskeleton. J Biomech 43:9-14. CrossRef Medline

Sun Y, BamjiSX (2011) beta-Pix modulates actin-mediated recruitment of synaptic vesicles to synapses. J Neurosci 31:17123-17133. CrossRef Medline

Uruno T, Liu J, Zhang P, Fan Yx, Egile C, Li R, Mueller SC, Zhan X (2001) Activation of Arp2/3 complex-mediated actin polymerization by cortactin. Nat Cell Biol 3:259-266. CrossRef Medline

van den Heuvel M, Harryman-Samos C, Klingensmith J, Perrimon N, Nusse
$\mathrm{R}$ (1993) Mutations in the segment polarity genes wingless and porcupine impair secretion of the wingless protein. EMBO J 12:5293-5302. Medline

Wayman GA, Impey S, Marks D, Saneyoshi T, Grant WF, Derkach V, Soderling TR (2006) Activity-dependent dendritic arborization mediated by CaM-kinase I activation and enhanced CREB-dependent transcription of Wnt-2. Neuron 50:897-909. CrossRef Medline

Weaver AM, Karginov AV, Kinley AW, Weed SA, Li Y, Parsons JT, Cooper JA (2001) Cortactin promotes and stabilizes Arp2/3-induced actin filament network formation. Curr Biol 11:370-374. Medline

Weaver AM, Heuser JE, Karginov AV, Lee WL, Parsons JT, Cooper JA (2002) Interaction of cortactin and N-WASp with Arp2/3 complex. Curr Biol 12:1270-1278. CrossRef Medline

Wragg RT, Snead D, Dong Y, Ramlall TF, Menon I, Bai J, Eliezer D, Dittman JS (2013) Synaptic vesicles position complexin to block spontaneous fusion. Neuron 77:323-334. CrossRef Medline

Yao J, Qi J, Chen G (2006) Actin-dependent activation of presynaptic silent synapses contributes to long-term synaptic plasticity in developing hippocampal neurons. J Neurosci 26:8137-8147. CrossRef Medline

Yu X, Malenka RC (2003) Beta-catenin is critical for dendritic morphogenesis. Nat Neurosci 6:1169-1177. CrossRef Medline 